\title{
Categorical symmetry and noninvertible anomaly in symmetry-breaking and topological phase transitions
}

\author{
Wenjie Ji 10 and Xiao-Gang Wen \\ Department of Physics, Massachusetts Institute of Technology, Cambridge, Massachusetts 02139, USA
}

(Received 31 December 2019; revised 3 June 2020; accepted 3 June 2020; published 15 September 2020)

\begin{abstract}
For a zero-temperature Landau symmetry-breaking transition in $n$-dimensional space that completely breaks a finite symmetry $G$, the critical point at the transition has the symmetry $G$. In this paper, we show that the critical point also has a dual symmetry-a $(n-1)$-symmetry described by a higher group when $G$ is Abelian or an algebraic $(n-1)$-symmetry beyond a higher group when $G$ is non-Abelian. In fact, any $G$-symmetric system can be viewed as a boundary of $G$-gauge theory in one higher dimension. The conservation of gauge charge and gauge flux in the bulk $G$-gauge theory gives rise to the symmetry and the dual symmetry, respectively. So any $G$-symmetric system actually has a larger symmetry called categorical symmetry, which is a combination of the symmetry and the dual symmetry. However, part (and only part) of the categorical symmetry must be spontaneously broken in any gapped phase of the system, but there exists a gapless state where the categorical symmetry is not spontaneously broken. Such a gapless state corresponds to the usual critical point of Landau symmetry-breaking transition. The above results remain valid even if we expand the notion of symmetry to include higher symmetries and algebraic higher symmetries. Thus our result also applies to critical points for transitions between topological phases of matter. In particular, we show that there can be several critical points for the transition from the $3+1$-dimensional $Z_{2}$ gauge theory to a trivial phase. The critical point from Higgs condensation has a categorical symmetry formed by a $Z_{2} \quad 0$-symmetry and its dual, a $Z_{2}$ 2-symmetry, while the critical point of the confinement transition has a categorical symmetry formed by a $Z_{2} \quad 1$-symmetry and its dual, another $Z_{2}$ 1-symmetry.
\end{abstract}

DOI: 10.1103/PhysRevResearch.2.033417

\section{INTRODUCTION}

Consider a Landau symmetry-breaking transition [1,2] in a quantum system in $n$-dimensional space at zero temperature that completely breaks a finite on-site symmetry $G$. The critical point at the transition is a gapless state with $G$ symmetry. When $n=1$, it is well known that the $1+1$ dimensional $(1+1 \mathrm{D})$ gapless critical point has two decoupled sectors at low energies: right movers and left movers [3,4]. Thus the critical point has a low-energy emergent symmetry $G \times G$. In this paper, we would like to show that a similar symmetry "doubling" phenomenon also appears for critical points of Landau symmetry-breaking phase transitions in all other dimensions. In this paper we will use $n \mathrm{D}$ to refer to space dimensions and $(n+1) \mathrm{D}$ to refer to spacetime dimensions.

In general, the quantum critical point always connects two $n \mathrm{D}$ phases: a symmetric phase with no ground-state degeneracy and a symmetry-breaking phase with $|G|$ degenerate ground states. The symmetric phase is characterized by its pointlike excitations that carry irreducible representations of

Published by the American Physical Society under the terms of the Creative Commons Attribution 4.0 International license. Further distribution of this work must maintain attribution to the author(s) and the published article's title, journal citation, and DOI.
$G$. The collection of those pointlike excitations, plus their trivial braiding and nontrivial fusion properties, give us a local fusion $n$-category denoted by $n \mathcal{R} \operatorname{ep}(G)$ (see, for example, Refs. [5-8] and Appendix A). The conservation of those pointlike excitations is encoded by the nontrivial fusion of the irreducible representations $R_{q}$ :

$$
R_{q_{1}} \otimes R_{q_{2}}=\bigoplus_{q_{3}} N_{q_{1} q_{2}}^{q_{3}} R_{q_{3}}
$$

which reflects the $G$ symmetry. In fact, due to Tannaka duality, the local fusion $n$-category $n \mathcal{R} \operatorname{ep}(G)$ completely characterizes the symmetry group $G$. Since the critical point touches the symmetric phase, the ground state at the critical point is also symmetric under $G$.

The symmetry-breaking phase has ground states labeled by the group elements $g \in G,\left|\Psi_{g}\right\rangle$, that are not invariant under the symmetry transformation: $U_{h}\left|\Psi_{g}\right\rangle=\left|\Psi_{h g}\right\rangle, g, h \in G$. We can always consider a symmetrized ground state $\left|\Psi_{0}\right\rangle=$ $\sum_{g}\left|\Psi_{g}\right\rangle$ that is invariant under the symmetry transformation: $U_{h}\left|\Psi_{0}\right\rangle=\left|\Psi_{0}\right\rangle$. The symmetry-breaking phase has domainwall (DW) excitations and we only consider the symmetrized states with domain walls. The domain walls can also fuse in a nontrivial way, and form a local fusion $n$-category, denoted by $n \mathcal{V} \mathrm{ec}_{G}$ (see Refs. [7,8] and Appendix A). The nontrivial fusion also leads to a "conservation" of domain-wall excitations. 
It turns out that the "conservation" of $(n-1)$-dimensional domain-wall excitations can be viewed as a result of algebraic higher symmetry [7,8] generated by closed string operators $W_{q}\left(S^{1}\right)$ that commute with the lattice Hamiltonian

$$
W_{q}\left(S^{1}\right) H=H W_{q}\left(S^{1}\right),
$$

for all the closed loops $S^{1}$ (see Sec. V). Reference [8] conjectured that there is a generalization of Tannaka duality: a local fusion $n$-category $\mathcal{R}$ ( $\operatorname{such}$ as $n \mathcal{V} \mathrm{ec}_{G}$ ) completely characterizes an algebraic higher symmetry [in the present case, an algebraic $(n-1)$-symmetry]. When the symmetry group is Abelian, such an algebraic $(n-1)$-symmetry is a $(n-1)$-symmetry described by a higher group. When the symmetry group is non-Abelian, such an algebraic $(n-1)$ symmetry is beyond higher group description and is not a $(n-1)$-symmetry.

To contrast higher symmetry (described by higher group) and algebraic higher symmetry (beyond higher group), we note that for grouplike higher symmetry the string operators satisfy a grouplike algebra

$$
W_{q_{1}}\left(S^{1}\right) W_{q_{2}}\left(S^{1}\right)=W_{q_{1} \cdot q_{2}}\left(S^{1}\right),
$$

while for algebraic higher symmetry, the string operators satisfy a more general algebra

$$
W_{q_{1}}\left(S^{1}\right) W_{q_{2}}\left(S^{1}\right)=\sum_{q_{3}} N_{q_{1} q_{2}}^{q_{3}} W_{q_{3}}\left(S^{1}\right) .
$$

The notion of algebraic higher symmetry has appeared in Refs. [9-13] for 1+1D conformal field theory (CFT) via noninvertible defect lines and for lattice models in general dimensions in Refs. [7,8]. This generalizes the notion of higher form symmetry [14-22] (or higher symmetry [23-29]). ${ }^{1} \mathrm{~A}$ higher symmetry is described by a higher group, while an algebraic higher symmetry is beyond higher groups. The charged excitations (the charge objects) of an algebraic higher symmetry are in general characterized by a local fusion higher category (see Ref. [8] and Appendix A).

We see that the $G$ symmetry-breaking phase, with domainwall excitations forming $n \mathcal{V e c}_{G}$, actually has an algebraic $(n-1)$-symmetry, denoted as $G^{(n-1)}$. Since the critical point touches the symmetry-breaking phase, the critical point also has the algebraic $(n-1)$-symmetry $G^{(n-1)}$. Therefore, the gapless state, at the critical point of $G$ symmetry-breaking transition, has both the $G$ zero symmetry and the algebraic $(n-1)$-symmetry $G^{(n-1)}$. We call this combined symmetry a categorical symmetry. In fact, the categorical symmetry even appears off the critical point, but in a spontaneously broken form.

There is a holographic way to see the appearance of categorical symmetry in any $G$-symmetric system. We note

\footnotetext{
${ }^{1}$ Higher form symmetry and higher symmetry are similar. They have only a small difference: The action of higher form symmetry becomes an identity when it acts on contractible closed subspaces, while the action of higher symmetry may not be an identity when it acts on contractible closed subspaces. Higher symmetry is a symmetry in a lattice model. Higher symmetry reduces to higher form symmetry in gapped ground-state subspaces (i.e., in the low-energy effective topological quantum field theory).
}

that when restricted to the symmetric sub-Hilbert space a $n \mathrm{D}$ $G$-symmetric system can be viewed as a system that has a noninvertible gravitational anomaly, i.e., can be viewed as the boundary of the $G$-gauge theory in one higher dimension [30,31]. The bulk gauge charges, when brought to the boundary, are the excitations of the $G 0$-symmetry, and the gauge fluxes, brought to the boundary, are excitations of the algebraic $(n-1)$-symmetry. The conservation of the gauge charges and gauge fluxes in $G$-gauge theory in the bulk leads to the $G 0$-symmetry and algebraic $(n-1)$-symmetry $G^{(n-1)}$, respectively, in our $G$-symmetric system that corresponds to the boundary (for details, see Sec. IID). Therefore, the $G$ symmetric system actually has a larger symmetry-the categorical symmetry, which is a combination of the symmetry (from the conservation of gauge charges) and the algebraic ( $n-1)$-symmetry (from the conservation of gauge flux), with nontrivial mutual statistics between gauge charges and gauge flux. Such a categorical symmetry is fully characterized by the $G$-gauge theory in one higher dimension.

Since the gapped boundaries of $G$-gauge theory always come from the condensation of the gauge charges and/or the gauge flux, the gapped ground state of the $G$-symmetric system always breaks the categorical symmetry partially, either the $G$ symmetry, or the algebraic $(n-1)$-symmetry, or some other mixtures of the two symmetries. A state with the full categorical symmetry [i.e., both the $G$ symmetry and the algebraic $(n-1)$-symmetry] must be gapless. We show that such a gapless state describes the critical point of the Landau symmetry-breaking transition.

More generally, all possible gapless states in a $G$ symmetric system are classified by gapless boundaries of $G$-gauge theory in one higher dimension. This universal emergence of categorical symmetry at the critical point, and its origin from a noninvertible gravitational anomaly (i.e., topological order in one higher dimension), may help us to systematically understand gapless states of matter.

It is worthwhile to point out that a structure similar to categorical symmetry was found previously in Antide Sitter/Conformal field theory (AdS/CFT) correspondence [32-35], where a global symmetry $G$ in a CFT at the boundary is related to an appearance of a gauge theory of group $G$ in the bulk. In this paper, we stress that the categorical symmetry encoded by the bulk is actually a bigger symmetry than the usual symmetry at the boundary. We point out that the $G$-symmetric gapless critical theory at the $(n+1) \mathrm{D}$ boundary actually has both the zero symmetry $G$ and the dual algebraic $(n-1)$-symmetry, which together form the categorical symmetry. For a more detailed discussion, see Sec. VIII.

In the following, we begin with studying a few concrete examples, to show the appearance of categorical symmetries in Landau symmetry-breaking transitions and in topological phase transitions, as well as neighboring gapped states that partially break the categorical symmetries spontaneously. In Secs. II-IV, we discuss the example of models associated with the $Z_{2}$ group in $1+1 \mathrm{D}$ and $2+1 \mathrm{D}$ cases without and with 't Hooft anomaly. We introduce the patch operators, as a main tool to detect local charges in sub-Hilbert space that is symmetric under global symmetries. We show how to use the patch operators to describe categorical symmetry. In Sec. V, we discuss the categorical symmetries in the 
lattice models with general finite group $G$ in any $n$ spatial dimensions. In Sec. VI, we discuss the emergence of algebraic higher symmetry. In Sec. VII, we discuss the emergence of categorical symmetry for a set of low-energy excitations, and how the categorical symmetry can constrain on the possible phases and phase transitions induced by those low-energy excitations. In particular, we discuss how the emergent categorical symmetry determines the duality relations between low-energy effective field theories. In Sec. VIII, we summarize our results and point out their implication for a particular AdS/CFT dual.

\section{II. $Z_{2}$ AND DUAL $\tilde{Z}_{2}$ SYMMETRIES IN THE $1+1 \mathrm{D}$ ISING MODEL}

\section{A. Duality transformation in the $1+1 D$ Ising model}

A common scenario happening at the critical point between the symmetric phase and the symmetry-breaking phase is the emergent symmetry. The example we know the best is the Ising transition in one dimension. The paramagnetic phase is $Z_{2}$ symmetric, and the ferromagnetic phase spontaneously breaks the $Z_{2}$ symmetry. The critical point is $Z_{2}$ symmetric. More than that, it also has an additional $Z_{2}$ symmetry $[11,36-$ 39].

To see both $Z_{2}$ symmetries, we consider an Ising model on a ring of $L$ sites, where on each site $i$ there are spin-up and spin-down states in the Pauli- $Z_{i}$ basis. So the Hilbert space is $\mathcal{V}=\otimes_{i=0}^{L-1}\left\{\left|\uparrow_{i}\right\rangle,\left|\downarrow_{i}\right\rangle\right\}$. Each state in the Hilbert space can be also labeled in an alternative way, that is, via the absence or presence of a DW in the dual lattice of $L$ links. On each link $i+\frac{1}{2}$, a domain wall means the spins on $i$ and $i+1$ are antiparallel. It follows that each basis state $|\psi\rangle$ of $\mathcal{V}$ and its $Z_{2}$ partner $\left|\psi^{\prime}\right\rangle$ are labeled by the same kink variable on the dual lattice. So the $Z_{2}$-symmetric state in $\mathcal{V}$ is labeled uniquely by the DW variable. Moreover, a configuration of an odd number of domain walls on links cannot be mapped to any configuration of spins on sites. Thus each DW variable with an even number of DW's labels a unique $Z_{2}$-symmetric state. Therefore, we say that the $Z_{2}$-symmetric Hilbert space of spins on the sites is in one-to-one correspondence with the Hilbert space of an even number of DWs on the links. Each Hilbert space is of dimension $2^{L-1}$.

Next, we demonstrate an isomorphism between a set of operators on sites and one on links:

$$
X_{i} I_{i+1} \rightarrow \widetilde{X}_{i-\frac{1}{2}} \widetilde{X}_{i+\frac{1}{2}}, \quad Z_{i} Z_{i+1} \rightarrow \widetilde{I}_{i-\frac{1}{2}} \widetilde{Z}_{i+\frac{1}{2}} .
$$

Here, we use the following notation:

$$
X=\left(\begin{array}{ll}
0 & 1 \\
1 & 0
\end{array}\right), \quad Z=\left(\begin{array}{cc}
1 & 0 \\
0 & -1
\end{array}\right), \quad I=\left(\begin{array}{ll}
1 & 0 \\
0 & 1
\end{array}\right) .
$$

Physically, the spin-flipping $X_{i}$ is the same as creating two DW's on $i-\frac{1}{2}$ and $i+\frac{1}{2}$ links, represented by $\widetilde{X}_{i-\frac{1}{2}} \widetilde{X}_{i+\frac{1}{2}}$. The Ising coupling term $Z_{i} Z_{i+1}$ also measures the energy cost of a domain wall, which is represented by $\widetilde{Z}_{i+\frac{1}{2}}$. Formally, the two sets of operators $\left\{X_{i}, Z_{i} Z_{i+1}\right\}$ and $\left\{\widetilde{X}_{i-\frac{1}{2}} \widetilde{X}_{i+\frac{1}{2}}, \widetilde{Z}_{i+\frac{1}{2}}\right\}$ are two representations of the same set of operators $\left\{W_{i}, W_{i+\frac{1}{2}}\right\}$ defined by the operator algebra, for $i=\frac{1}{2}, 1, \cdots, L$ :

(i) $W_{i}^{2}=1$,

(ii) $W_{i} W_{i+\frac{1}{2}}=-W_{i+\frac{1}{2}} W_{i}$,

(iii) $\left[W_{i}, W_{j}\right]=0,|i-j| \geqslant 1$.

We also have a further global condition:

$$
\begin{aligned}
U_{Z_{2}} & =\prod_{i=1,2, \cdots, L} W_{i}=1, \\
U_{\widetilde{Z}_{2}} & =\prod_{i=\frac{1}{2}, \frac{3}{2}, \cdots L-\frac{1}{2}} W_{i}=1 .
\end{aligned}
$$

We have two $2^{L-1}$ dimensional representations of $W_{i}$ 's, satisfying the relations (7) and (8). In particular, the following Hamiltonian,

$$
H=-\sum_{i=1,2 \cdots, L}\left(B W_{i}+J W_{i+\frac{1}{2}}\right),
$$

has the same spectrum independent of the representation. In the "spin representation," the Hamiltonian reduces to

$$
\begin{aligned}
H^{\mathrm{Is}} & =-\sum_{i=1,2, \cdots, L}\left(B X_{i}+J Z_{i} Z_{i+1}\right), \\
U_{Z_{2}} & =\prod_{i=1,2, \cdots L} X_{i}=1, \quad U_{\widetilde{Z}_{2}}=Z_{L+1} Z_{1}=1 .
\end{aligned}
$$

In the "DW representation," the Hamiltonian reduces to

$$
\begin{aligned}
H^{\mathrm{DW}} & =-\sum_{i=1,2, \cdots, L}\left(B \widetilde{X}_{i-\frac{1}{2}} \widetilde{X}_{i+\frac{1}{2}}+J \widetilde{Z}_{i+\frac{1}{2}}\right), \\
U_{Z_{2}} & =\widetilde{X}_{L+\frac{1}{2}} \widetilde{X}_{\frac{1}{2}}=1, \quad U_{\widetilde{Z}_{2}}=\prod_{i=1,2, \cdots L} \widetilde{Z}_{i-\frac{1}{2}}=1 .
\end{aligned}
$$

The unitary transformation (5) between the spin representation and DW representation is also known as $Z_{2}$ gauging. In the current case, it is also the same as Kramers-Wannier duality.

The Ising model has two exact $Z_{2}$ symmetries. However, in our spin and DW representations, we do not see them simultaneously. In the spin representation, we see one $Z_{2}$ symmetry generated by

$$
U_{Z_{2}}=\prod_{i=1, \cdots, L} X_{i},
$$

which is denoted as $Z_{2}$. In the DW representation, we see the other $Z_{2}$ symmetry generated by

$$
U_{\widetilde{Z}_{2}}=\prod_{i=0, \cdots, L} \widetilde{Z}_{i+\frac{1}{2}},
$$

which is denoted as $\widetilde{Z}_{2} . Z_{2}$ and $\widetilde{Z}_{2}$ are two different $Z_{2}$ symmetries, as one can see from their different charge excitations. Despite the fact that we only see one symmetry in one formulation, the Ising model actually has both symmetries. The combination of the two symmetries is the so-called categorical symmetry, which is denoted as $Z_{2} \vee \widetilde{Z}_{2}$. Certainly, the critical model at $|J|=|B|$ also has the $Z_{2} \vee \widetilde{Z}_{2}$ categorical symmetry (see Fig. 1). It is interesting to note that, in the ground state of the Ising model, either $Z_{2} \vee \widetilde{Z}_{2}$ categorical symmetry is 


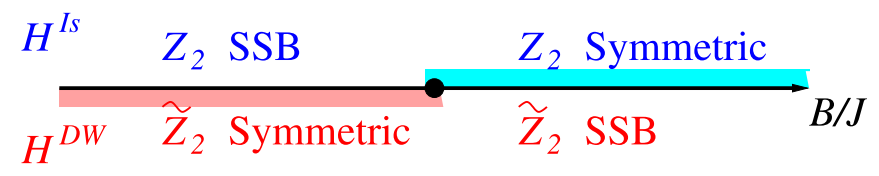

FIG. 1. The same Ising model can be described by $H^{\mathrm{Is}}$ or by $H^{\text {DW }}$. The $Z_{2}$ symmetry is explicit in the $H^{\text {Is }}$ description, while the $\widetilde{Z}_{2}$ dual symmetry is explicit in the $H^{\mathrm{DW}}$ description. The Ising model has both the $Z_{2}$ symmetry and $\widetilde{Z}_{2}$ dual symmetry. The ground state usually spontaneously breaks one of the symmetries, except at the $B=J$ critical point, where both the symmetry and the dual symmetry (i.e., the full $Z_{2} \vee \widetilde{Z}_{2}$ categorical symmetry) are not spontaneously broken.

spontaneously broken partially (for example, one of the $Z_{2}$ is spontaneously broken) or the ground state is gapless [40]. This indicates that $Z_{2}$ symmetry and the dual $\widetilde{Z}_{2}$ symmetry are not independent. There must be a special relation between them. To reveal it, we need to discuss the charge excitations of the symmetries.

\section{B. Patch symmetry transformation}

In the above, we argue that the lattice model Eq. (9) [or Eq. (10) or Eq. (11)] in the symmetric sub-Hilbert space has two $Z_{2}$ symmetries generated by $U_{Z_{2}}$ and $U_{\widetilde{Z}_{2}}$. But in the symmetric sub-Hilbert space, the two operators are identity operator $U_{Z_{2}}=U_{\widetilde{Z}_{2}}=1$. The two $Z_{2}$ transformations are do-nothing transformations. So what does it mean that the lattice model in symmetric sub-Hilbert space has two $Z_{2}$ symmetries? In this section, we are going to solve this problem by introducing patch symmetry transformations. Such kind of operators has been studied mostly for continuous global symmetries, known in the literature [41] as splittable symmetry operators, dating back to the 1980s [42-44].

We notice that even though $U_{Z_{2}}$ and $U_{\widetilde{Z}_{2}}$ act as identities in the symmetric sub-Hilbert space the sub-Hilbert space does not consist of only a vacuum state. Rather, spin-flip as well as domain-wall excitations are still present, and they are subject to mod- 2 conservations. So the effect of two $Z_{2}$ symmetries is still there within the symmetric sub-Hilbert space. For example, there exists a state on the ring containing two wellseparated spin excitations, each carrying the $U_{Z_{2}}$ charge 1 while the total $U_{Z_{2}}$ charge is zero mod 2 . How do we measure the local $U_{Z_{2}}$ charge via a symmetry transformation operator?

To diagnose the conserved local charges of $U_{Z_{2}}$ and $U_{\widetilde{Z}_{2}}$ symmetries, subject to conservation laws, we now introduce two sets of patch symmetry operators ${ }^{2}$ for the simple model Eq. (10) or Eq. (11).

The first patch symmetry is generated by the following transformations:

$$
U_{Z_{2}}(i, j)=\prod_{l=i}^{j} X_{l}, \text { for } j-i \geqslant 0,
$$

which are required to act within the symmetric sub-Hilbert space. They also have the properties that for any $j-i \geqslant$

\footnotetext{
${ }^{2}$ We hope the name is intuitive even when generalized to higher dimensions.
}

$$
\begin{aligned}
1, j^{\prime}-i^{\prime} \geqslant 0 & \\
U_{Z_{2}}(i, k) & =U_{Z_{2}}(i, j) U_{Z_{2}}(j+1, k), \\
U_{Z_{2}}(i, j)^{2} & =1, \quad\left[U_{Z_{2}}(i, j), U_{Z_{2}}\left(i^{\prime}, j^{\prime}\right)\right]=0 .
\end{aligned}
$$

In condensed-matter physics, a symmetry is simply a constraint on the lattice Hamiltonian $H$. We usually describe such a constraint as a constraint on the Hamiltonian $H$ as a whole. Under such constraint, the Hamiltonian $H$ is allowed to be local or nonlocal. This actually is a drawback of the standard formulation of the symmetry, since it does not care about the locality of the Hamiltonian. In our description of symmetry, using patch operators, we assume the Hamiltonian $H$ to be sum of local operators $H=\sum_{x} O_{x}$. Then the constraint on the Hamiltonian $H$ is expressed in terms of the constraint on the local terms $O_{x}$. In other words, a system is said to have the patch symmetry, if it has the following properties: each local term in the Hamiltonian commutes with all the patch symmetry operators, as long as the local term is far away from the boundary of the patch operator. For example, if $H_{l}$ is a term on $l, l+1$, and if it commutes with all patch symmetry operators $U_{Z_{2}}(i, j)$ with $i, j \neq l, l+1$, this term is symmetric under the patch symmetry. If every term in the Hamiltonian has this property, we say the Hamiltonian has the patch symmetry.

Each patch symmetry operator $U_{Z_{2}}(i, j)$ measures the $Z_{2}$ spin excitations in its "bulk," the sites covered by the patch, from site $i$ to site $j$. More precisely, a local operator $\psi_{k}$ carries $Z_{2}$ charge 1 if it satisfies

$$
U_{Z_{2}}(i, j) \psi_{k}=-\psi_{k} U_{Z_{2}}(i, j), \quad i \ll k \ll j .
$$

$\psi_{k} \psi_{l}$ creates two $Z_{2}$ charges at $k$ and $l$ within the symmetric sub-Hilbert space.

In Eq. (14), the patch symmetry operator is given in the spin representation. In the DW representation, it will have the form

$$
U_{Z_{2}}(i, j)=\tilde{X}_{i-\frac{1}{2}} \tilde{X}_{j+\frac{1}{2}}
$$

which has a trivial "body of the string" but only two end points. By the definition given above about a system symmetric under the patch symmetry, and due to the trivial bulk, any local Hamiltonian in the DW representation is symmetric under $U_{Z_{2}}(i, j)$. We may also take the patch operators $U_{Z_{2}}(i, j)$ as creating a pair of $\widetilde{Z}_{2}$-charged excitations (i.e., a pair of $Z_{2}$ domain walls) at the ending links $i-\frac{1}{2}$ and $j+\frac{1}{2}$.

The second patch symmetry is generated by the following operators, in the spin and DW representations:

$$
U_{\widetilde{Z}_{2}}(i, j)=Z_{i} Z_{j}, \quad U_{\widetilde{Z}_{2}}(i, j)=\prod_{l=i}^{j-1} \widetilde{Z}_{l+\frac{1}{2}},
$$

which have the properties that they act within the symmetric sub-Hilbert space, for any $j-i \geqslant 1, j^{\prime}-i^{\prime} \geqslant 1$ :

$$
\begin{aligned}
U_{\widetilde{Z}_{2}}(i, k) & =U_{\widetilde{Z}_{2}}(i, j) U_{\widetilde{Z}_{2}}(j, k), \\
U_{\widetilde{Z}_{2}}(i, j)^{2} & =1, \quad\left[U_{\widetilde{Z}_{2}}(i, j), U_{\widetilde{Z}_{2}}\left(i^{\prime}, j^{\prime}\right)\right]=0 .
\end{aligned}
$$

We see that any local Hamiltonian in spin representation has the $U_{\widetilde{Z}_{2}}$ symmetry. However, for the Hamiltonian in the DW representation, the second patch symmetry gives rise to 
a nontrivial constraint on the Hamiltonian. These symmetry operators serve to measure local domain-wall excitations. We can see this in the spin representation: when $Z_{i}$ and $Z_{j}$ have opposite sign, i.e., there is a domain between $i$ and $j$, then $U_{\widetilde{Z}_{2}}(i, j)=Z_{i} Z_{j}=-1$.

In summary, we identify two patch symmetries, each of which is generated by a set of commuting operators. The two kinds of patch symmetries act nontrivially even in the symmetric sub-Hilbert space, and can impose constraints on Hamiltonians. The symmetric Hamiltonians ensure the mod-2 conservation of the $Z_{2}$ charges and domain walls.

The patch symmetry transformations also allow us to identify a special new property-the "mutual statistics" between charges of the two global symmetries (or patch symmetries), given by the following relation, for $i \ll i^{\prime} \ll j \ll j^{\prime}$ :

$$
U_{Z_{2}}(i, j) U_{\widetilde{Z}_{2}}\left(i^{\prime}, j^{\prime}\right)=-U_{\widetilde{Z}_{2}}\left(i^{\prime}, j^{\prime}\right) U_{Z_{2}}(i, j) .
$$

Alternatively, a local charge under the $Z_{2}$ global symmetry is created at each end point of a $\widetilde{Z}_{2}$ patch operator. If a $Z_{2}$ symmetry patch and a $\widetilde{Z}_{2}$ symmetry patch partially overlap, the single charge at one end point can be measured by the $Z_{2}$ patch symmetry operator. All such statements remain true if exchanging $Z_{2}$ and $\widetilde{Z}_{2}$. We call such properties the mutual statistics between charges of the two patch symmetries. The collection of all patch symmetries is nothing but the categorical symmetry. The property (20) justifies the symmetry to be $Z_{2} \vee \widetilde{Z}_{2}$, rather than $Z_{2} \times \widetilde{Z}_{2}$.

We see that the categorical symmetry in a system can be fully described by a set of patch operators, without the need to go to one higher dimension. Alternatively, later in Sec. II D, we describe the categorical symmetry in terms of the topological order and the associated long-range entanglement, by viewing the system as a boundary of a topological order in one higher dimension. The above result confirms that the categorical symmetry is indeed a property of the system itself.

Note that $\left\langle U_{\widetilde{Z}_{2}}(i, j)\right\rangle$ also turns out to be the correlation of order parameters of the $Z_{2}$ symmetry, while $\left\langle U_{Z_{2}}(i, j)\right\rangle$ happens to be the correlation of order parameters of the $\widetilde{Z}_{2}$ symmetry. If there is a state, where both the $Z_{2}$ symmetry and the $\widetilde{Z}_{2}$ symmetry are spontaneously broken, then $U_{\widetilde{Z}_{2}}(i, j)$ and $U_{Z_{2}}(i, j)$ behave like complex numbers for the state. This will contradict with Eq. (20). Therefore, the $Z_{2}$ symmetry and the $\widetilde{Z}_{2}$ symmetry cannot be both spontaneously broken. A more rigorous proof was given in Ref. [40].

\section{A model where both $Z_{2}$ symmetry and dual $\tilde{Z}_{2}$ symmetry are explicit}

The Ising model in its spin representation Eq. (10) or in its DW representation Eq. (11) only shows one of the $Z_{2}$ and dual $\widetilde{Z}_{2}$ symmetries explicitly. In this section, we will discuss the third representation of the Ising model, where both the $Z_{2}$ and dual $\widetilde{Z}_{2}$ symmetries appear explicitly.

Consider a model with spin-up and down states defined on $N$ sites as well as on $N$ links. So we begin with $2^{2 N}$ states. The model has the following Hamiltonian:

$$
\begin{aligned}
H= & -\sum_{i}\left(B \widetilde{X}_{i-\frac{1}{2}} X_{i} \widetilde{X}_{i+\frac{1}{2}}+J \widetilde{Z}_{i+\frac{1}{2}}\right) \\
& +U\left(1-Z_{i} \widetilde{Z}_{i+\frac{1}{2}} Z_{i+1}\right) .
\end{aligned}
$$

We only consider the low-energy limit $U \rightarrow \infty$, as well as the projection $U_{Z_{2}}=\prod_{i} X_{i}=1$. (Note that the $B$ term and the $J$ term commute with the constraint $U$ term and $U_{Z_{2}}$.) In the restricted low-energy sector, we are left with $2^{N-1}$ states. The above Hamiltonian is conventionally known as describing the $Z_{2}$ matter field coupled to the $Z_{2}$ gauge field in the $1+1 \mathrm{D}$ dual lattice. ${ }^{3}$ The Ising model (10) with the $Z_{2}$-symmetric sub-Hilbert space turns out to be equivalent as the low-energy effective theory of the above model with $B, J>0$, and $U \rightarrow+\infty$. There is more than one way to prove this. In Appendix B, we give a proof using the stabilizer formalism in quantum information. In the next subsection, we will show that the same Hamiltonian together with the same projected sub-Hilbert space arises as the boundary theory of the $Z_{2}$ topological order.

The $Z_{2} \times \widetilde{Z}_{2}$ symmetry (or more precisely, the $Z_{2} \vee \widetilde{Z}_{2}$ categorical symmetry) is explicit in the above model, which is generated by $U_{Z_{2}}$ in Eq. (12) and $U_{\widetilde{Z}_{2}}$ in Eq. (13) as an on-site symmetry of the model. Even though the $Z_{2} \times \widetilde{Z}_{2}$ symmetry is on site in the model (21), it is anomalous when restricted in the low-energy sector in the sense that in the $U=+\infty$ limit the model cannot have a gapped ground state that breaks the full $Z_{2} \times \widetilde{Z}_{2}$ symmetry. (Certainly, when $U<J, B$, the model can have a gapped ground state that breaks the full $Z_{2} \times \widetilde{Z}_{2}$ symmetry, such as when $J=B=1, U=0$.) In the $U=+\infty$ limit, only the gapless state at $J=B$ has the full $Z_{2} \times \widetilde{Z}_{2}$ symmetry that is not spontaneously broken.

What are the patch symmetry transformations for the $Z_{2}$ and $\widetilde{Z}_{2}$ symmetry? The first guesses are

$$
U_{Z_{2}}(i, j)=\prod_{k=i}^{j} X_{k}, \quad U_{\widetilde{Z}_{2}}(i, j)=\prod_{k=i}^{j} \widetilde{Z}_{k+\frac{1}{2}} .
$$

But $U_{Z_{2}}(i, j)=\prod_{k=i}^{j} X_{k}$ does not act within the low-energy sub-Hilbert space (in the $U=+\infty$ limit). To get around this, we modify it at the boundary, which leads to

$$
\begin{aligned}
& U_{Z_{2}}(i, j)=\widetilde{X}_{i-\frac{1}{2}}\left(\prod_{k=i}^{j} X_{k}\right) \widetilde{X}_{j+\frac{1}{2}}, \\
& U_{\widetilde{Z}_{2}}(i, j)=\left(\prod_{k=i}^{j} \widetilde{Z}_{k+\frac{1}{2}}\right) .
\end{aligned}
$$

The two sets of patch symmetry transformations still satisfy the algebras Eqs. (15) and (19) as previously. Most importantly, the two sets of patch symmetry transformations have a $\pi$ mutual statistics described by Eq. (20). It is this property of the patch operators that we mean when we say that the symmetry we consider is $Z_{2} \vee \widetilde{Z}_{2}$, but not $Z_{2} \times \widetilde{Z}_{2}$. One can also easily check that the local terms in the Hamiltonian (21) commute with the patch symmetry transformations when far away from the boundary. So the Hamiltonian (21) has the $Z_{2}$ and $\widetilde{Z}_{2}$ patch symmetries. In short, although the global

\footnotetext{
${ }^{3}$ Here, $\widetilde{Z}_{i+\frac{1}{2}}$ and $\widetilde{X}_{i+\frac{1}{2}}$ are the matter field and momenta on the sites of the dual lattice. $Z_{i}$ and $X_{i}$ are the gauge field and momenta on the links of the dual lattice. The Gauss law $Z_{i} \widetilde{Z}_{i+\frac{1}{2}} Z_{i+1}=1$ is imposed as a dynamical constraint.
} 
symmetry transformations of $Z_{2}$ and $\widetilde{Z}_{2}$ commute, the patch operators, which create charges at their end points, do not commute in the low-energy sub-Hilbert-space limit when $U \rightarrow \infty$.

We would like to remark that the same symmetry can be described by different yet equivalent choices of patch symmetry transformations. Two sets of patch symmetry transformations are equivalent if the patch symmetry transformations only differ by "local neutral operators" at the boundary of the patches. Here a local neutral operator is a local operator that commutes with all patch symmetry transformations the boundary of which is far away from the operator. The mutual statistics of the patch symmetry transformations is not affected by those local neutral operators. For example, we may instead choose $U_{Z_{2}}(i, j)=\widetilde{Y}_{i-\frac{1}{2}}\left(\prod_{k=i}^{j} X_{k}\right) \widetilde{Y}_{j+\frac{1}{2}}$ in (23).

\section{Symmetric sector of the $1+1 D$ Ising model as the boundary of $2+1 \mathrm{D} Z_{2}$ topological order}

In the previous section, we discuss the anomaly property of $Z_{2} \vee \widetilde{Z}_{2}$ categorical symmetry, the mutual statistics of $Z_{2}$ and $\widetilde{Z}_{2}$ charges in the low-energy limit. This forbids a symmetric gapped ground state within the low-energy sector. In this section, we will show that the anomaly property of the $Z_{2} \vee \widetilde{Z}_{2}$ categorical symmetry is actually an effect of a noninvertible gravitational anomaly [31]. More precisely, the theory with the categorical symmetry can be a boundary theory of a $Z_{2}$ topological order in one higher dimension. The charges and their mutual statistics Eq. (20) of the symmetry is determined by the bulk topological order. To see the noninvertible gravitational anomaly in the $1+1 \mathrm{D}$ Ising model, we concentrate on the so-called symmetric sub-Hilbert space $\mathcal{V}_{\text {symm }}$ that is invariant under the $U_{Z_{2}}$ transformation. The space $\mathcal{V}_{\text {symm }}$ of a $L$-site system does not have tensor product expansion of the form $\otimes_{i=1}^{L} \mathcal{V}_{i}$ :

$$
\mathcal{V}_{\text {symm }} \neq \otimes_{i=1}^{L} \mathcal{V}_{i}
$$

Thus the symmetric sector of the Ising model can be viewed as having a noninvertible gravitational anomaly [31]. Indeed, the symmetric sector of the Ising model can be viewed as a boundary of $2+1 \mathrm{D} Z_{2}$ topological order (the topological order characterized by $Z_{2}$ gauge theory), and thus has a $1+1 \mathrm{D}$ noninvertible gravitational anomaly characterized by $2+1 \mathrm{D} Z_{2}$ topological order [31].

A $2+1 \mathrm{D} Z_{2}$ topological order has four types of excitations $\mathbb{1}, e, m$, and $f$. Here $\mathbb{1}$ is the trivial excitation, and $e, m$, and $f$ are topological excitations with mutual $\pi$ statistics between any two different ones. $\mathbb{1}, e$, and $m$ are bosons and $f$ is a fermion. They satisfy the following fusion relations:

$$
e \otimes e=\mathbb{1}, \quad m \otimes m=\mathbb{1}, \quad f \otimes f=\mathbb{1}, \quad e \otimes m=f .
$$

Let us construct the boundary effective theory for the $m$ condensed boundary of $Z_{2}$ topological order. Such a boundary contains a gapped excitation that corresponds to the $e$ type particle. One might expect a second boundary excitation corresponding to the $f$-type particle. However, since $m$ is condensed on the boundary, the $e$-type particle and the $f$-type particle are actual equivalent on the boundary. The simplest boundary effective lattice Hamiltonian that describes the gapped $e$-type particles has the form (on a ring)

$$
H=-B \sum_{i} X_{i}, \quad B>0 .
$$

Here a spin $X_{i}=1$ corresponds to an empty site and a spin $X_{i}=-1$ corresponds to a site occupied with an $e$-type particle (with $2 B$ as its energy gap). However, the boundary Hilbert space does not have a direct product decomposition $\otimes_{i} \mathcal{V}_{i}$, due to the constraint

$$
\prod_{i} X_{i}=1,
$$

since the number of the $e$-type particles on the boundary must be even (assume the bulk has no topological excitations). This is a reflection of a noninvertible gravitational anomaly. A more general boundary effective theory may have the form

$$
H_{P}^{\mathrm{Is}}=-B \sum_{i=1}^{L} X_{i}-J \sum_{i=1}^{L} Z_{i} Z_{i+1},
$$

where $Z_{L+1} \equiv Z_{1}$ and $Z_{i} Z_{i+1}$ creates a pair of the $e$-type particles, or moves an $e$-type particle from one site to another.

In the above, we have shown that a boundary of $2+$ $1 \mathrm{D} Z_{2}$ topological order can be described by Eq. (28). The low-energy sector of the model Eq. (21) also describes the boundary of the $2+1 \mathrm{D} Z_{2}$ gauge theory with $Z_{2}$ charge $e$ and $Z_{2}$ vortex $m$, where $e$ and $m$ particles have low energies on the boundary. An $e$ particle on the boundary corresponds to $X_{i}=-1$ and a $m$ particle corresponds to $\widetilde{Z}_{i+\frac{1}{2}}=-1$ in Eq. (21). The $Z_{2} \times \widetilde{Z}_{2}$ symmetry is the mod- 2 conservation of $e$ and $m$ particles. This explains the $Z_{2} \times \widetilde{Z}_{2}$ symmetry in the symmetric sector of the Ising model. Note that, on the boundary, we may have a $e$ or $m$ condensation. The condensations may spontaneously break the $Z_{2} \times \widetilde{Z}_{2}$ symmetry in the ground state. However, the model itself (given by the Hamiltonian and the sub-Hilbert space) always has $Z_{2} \times \widetilde{Z}_{2}$ symmetry.

It is more precise to refer to the $Z_{2} \times \widetilde{Z}_{2}$ symmetry as $Z_{2} \vee \widetilde{Z}_{2}$ categorical symmetry. This is because the $Z_{2}$ and $\widetilde{Z}_{2}$ symmetries are not independent. The $Z_{2}$ charge (the $e$ particle) and the $\widetilde{Z}_{2}$ charge (the $m$ particle) have a $\pi$ mutual statistics, when viewed as particles in one higher dimension. This gives rise to Eq. (20). The term $Z_{2} \vee \widetilde{Z}_{2}$ categorical symmetry includes such nontrivial mutual statistics between the $Z_{2}$ and $\widetilde{Z}_{2}$ symmetry.

The mutual $\pi$ statistics between $e$ and $m$ in the $2+1 \mathrm{D}$ bulk is encoded at the boundary by requiring the $Z_{2}$ domain wall to carry $\widetilde{Z}_{2}$ charge and the $\widetilde{Z}_{2}$ domain wall to carry $Z_{2}$ charge. This nontrivial mutual statistics has a highly nontrivial effect: in a gapped ground state, one and only one of $Z_{2}$ and $\widetilde{Z}_{2}$ symmetries must be spontaneously broken [40]. Thus, a symmetric state that does not break the $Z_{2} \vee \widetilde{Z}_{2}$ categorical symmetry must be gapless. This is a consequence of a $1+1 \mathrm{D}$ noninvertible gravitational anomaly [31] characterized by $2+$ $1 \mathrm{D} Z_{2}$ topological order (i.e., $Z_{2}$ gauge theory).

To summarize, in the above, we considered the boundary of $2+1 \mathrm{D} Z_{2}$ topological order. We argued that a boundary (gapped or gapless), as a system (with the symmetric subHilbert space), always has a $Z_{2} \vee \widetilde{Z}_{2}$ categorical symmetry. In contrast, a gapped boundary, as a state, has a partially spontaneous broken categorical symmetry, while one of the 


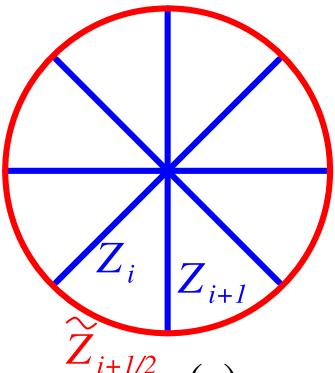

(a)

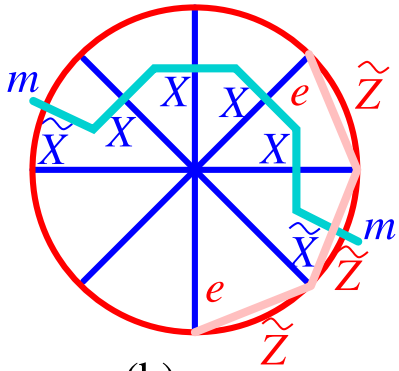

(b)
FIG. 2. The reduced lattice, where spin- $\frac{1}{2}$ degrees of freedom live on the links.

gapless boundaries, as a state, has the full categorical symmetry. Next, let us discuss patch symmetry transformations for the boundary of $2+1 \mathrm{D} Z_{2}$ topological order, that describe the $Z_{2} \vee \widetilde{Z}_{2}$ categorical symmetry.

We start with the bulk Hamiltonian for the $Z_{2}$ topological order on a square lattice, where spin- $\frac{1}{2}$ degrees of freedom live on the links:

$$
H^{\text {bulk }}=-U \sum_{s} \prod_{s \subset \partial l} X_{l}-U \sum_{p} \prod_{l \subset \partial p} Z_{l},
$$

where $s$ labels the sites, $l$ labels the links, and $p$ labels the plaquettes. $\sum_{s}$ sums over all the sites in the bulk (i.e., off the boundary), and $\sum_{p}$ sums over all the plaquettes. On the boundary, we can have any local Hamiltonian $H^{\text {bdy }}$. The boundary Hamiltonian remains finite as we take the $U \rightarrow \infty$ limit. $H^{\text {bulk }}$ in the $U \rightarrow \infty$ limit is a fixed-point Hamiltonian, and we can simplify it via tensor network renormalization [45-48]. In the end, the model Eq. (29) can be reduced to the one on the lattice in Fig. 2(a) [49]. And the Hamiltonian is still given by Eq. (29), which describes the dynamics of boundary degrees of freedom.

Now we will show that the wheel model with the Hamiltonian in Eq. (29) on a lattice [Fig. 2(a)] (plus extra boundary terms) and the sub-Hilbert space with no bulk excitations is the same as the minimally coupled model with the Hamiltonian in the symmetric sub-Hilbert space satisfying $\prod_{i} X_{i}=1$.

We consider the $Z_{2}$ topological order on the wheel, the fixed-point lattice of a disk. There are in total $2 N$ links, $N$ on the boundary and $N$ inside. That is in total $2^{2 N}$ states to start with. We consider the subspace that has no bulk excitations. That means the star and plaquette term satisfy

$$
\prod_{j=1}^{N} X_{j}=1, \quad Z_{i} \widetilde{Z}_{i+\frac{1}{2}} Z_{i+1}=1,
$$

for $i=1, \ldots, N$. These reduce the Hilbert space we consider to be of dimension $2^{N-1}$. Now we consider the boundary Hamiltonian. Any term in it should first commute with Eq. (30). Second, it describes the dynamics of $e$ and $m$ excitations on the boundary. In particular, we have

$$
H^{\text {bdy }}=-B \sum_{i} \tilde{X}_{i-\frac{1}{2}} X_{i} \widetilde{X}_{i+\frac{1}{2}}-J \sum_{i} \widetilde{Z}_{i}
$$

where the first term create pairs of $m$ particles, and the second term create pairs of $e$ particles. We may as well write the no bulk flux excitation as a dynamical constraint, and the boundary Hamiltonian is

$$
\begin{aligned}
H^{\mathrm{bdy}}= & -B \sum_{i} \widetilde{X}_{i-\frac{1}{2}} X_{i} \widetilde{X}_{i+\frac{1}{2}}-J \sum_{i} \widetilde{Z}_{i} \\
& +U \sum_{i}\left(1-Z_{i} \widetilde{Z}_{i+\frac{1}{2}} Z_{i+1}\right) .
\end{aligned}
$$

And this is the same as Eq. (21).

The upshot is that the $Z_{2}$ minimally coupled model with a global $Z_{2}$ constraint is equivalent to a boundary Hamiltonian of $\mathbb{Z}_{2}$ topological order when the bulk has no topological excitations. The ground-state subspace projection from the bulk to the boundary Hamiltonian is the same as the gauge and global constraint to the 1+1D minimally coupled model. ${ }^{4}$

Furthermore, one could see that the patch symmetry transformation $U_{Z_{2}}(i, j)$ [see Eq. (23)] is the same as the string operator that creates a pair of $e$-type excitations at string ends [see Fig. 2(b)], and the patch symmetry transformation $U_{\widetilde{Z}_{2}}(i, j)$ [see Eq. (23)] is the same as the string operator that creates a pair of $m$-type excitations at string ends [see Fig. 2(b)]. The excitations are only on the boundary of the wheel. This explains the nontrivial mutual statistics between $Z_{2}$ and $\widetilde{Z}_{2}$ patch symmetries, since the two string operators in Fig. 2(b) intersect at one point.

To summarize, a theory with a noninvertible gravitational anomaly has emergent symmetries (i.e., the categorical symmetry), which come from the conservation of topological excitations in one-higher-dimension bulk. Thus the categorical symmetry is fully characterized by the bulk topological order. Part of the categorical symmetry must be broken in any gapped phase, and the symmetric phase must be gapless. There is a gapless phase that respects the full categorical symmetry.

\section{E. How categorical symmetry determines the gapless state}

Equation (28) is a $m$-condensed boundary of $2+1 \mathrm{D} Z_{2}$ topological order. Its partition function has four components. For such a $m$-condensed boundary (with $|J|<B$ ), the fourcomponent partition function is given by (after shifting the ground-state energy density to zero) [31]

$$
\left(\begin{array}{l}
Z_{1} \\
Z_{e} \\
Z_{m} \\
Z_{f}
\end{array}\right)=\left(\begin{array}{l}
1 \\
0 \\
1 \\
0
\end{array}\right)
$$

Here

$$
Z_{1}=\operatorname{Tr}_{U_{Z_{2}}=1} e^{-\beta H_{P}^{\mathrm{Is}}}=1
$$

\footnotetext{
${ }^{4}$ In fact, take the $J=0$ limit of either the model Eq. (21) or the wheel model restricted to the boundary Eq. (32). The ground state is a SPT protected by $Z_{2} \times \widetilde{Z}_{2}$. However, under the $Z_{2} \vee Z_{2}$ symmetry specified by the patch operators Eq. (23), the ground state is at the same time a $m$ condensed state. This is because the patch operator $U_{Z_{2}}$ takes a constant value in the ground state. This is the string operator creating a pair of $m$, as illustrated in Fig. 2. Thus, the phase spontaneously breaks $Z_{2} \vee \widetilde{Z}_{2}$ symmetry.
} 
in the large $\beta, L$ limit and in the $U_{Z_{2}}=\prod_{i} Z_{i}=1$ sector. Also

$$
Z_{e}=\operatorname{Tr}_{U_{Z_{2}}=-1} e^{-\beta H_{P}^{\mathrm{Is}}}=0,
$$

in the large $\beta, L$ limit and in the $U_{Z_{2}}=\prod_{i} Z_{i}=-1$ sector. Similarly

$$
\begin{gathered}
Z_{m}=\operatorname{Tr}_{U_{Z_{2}}=1} e^{-\beta H_{A}^{\text {Is }}}=1, \\
Z_{f}=\operatorname{Tr}_{U_{Z_{2}=-1} e^{-\beta H_{A}^{\text {Is }}}=0,}
\end{gathered}
$$

where $H_{A}^{\mathrm{Is}}$ is the model Eq. (28) with an "antiperiodic boundary condition":

$$
H_{A}^{\mathrm{Is}}=-B \sum_{i=1}^{L} X_{i}-J \sum_{i=1}^{L-1} Z_{i} Z_{i+1}+J Z_{L} Z_{1},
$$

i.e., the $e$-type particle moving around the ring sees a $\pi$ flux.

The above partition functions describe a $Z_{2}$-symmetric gapped state. $Z_{e}=0$ implies the excitation carrying $Z_{2}$ charge to have a finite energy gap. $Z_{m}=1$ implies that the $Z_{2}$ symmetry is not spontaneously broken, since the $Z_{2}$ symmetry twist has no effect on the ground state. Also $Z_{m}=1$ means that the excitation carrying $Z_{2}$ flux has no energy gap. It also means that the patch symmetry operator $U_{Z_{2}}(i, j)$ (creating a pair of $Z_{2}$ flux excitations) has a nonzero average, i.e., the $\widetilde{Z}_{2}$ symmetry is spontaneously broken.

When $J>B$, we obtain the second gapped boundary:

$$
\left(\begin{array}{l}
Z_{1}(\tau, \bar{\tau}) \\
Z_{e}(\tau, \bar{\tau}) \\
Z_{m}(\tau, \bar{\tau}) \\
Z_{f}(\tau, \bar{\tau})
\end{array}\right)=\left(\begin{array}{l}
1 \\
1 \\
0 \\
0
\end{array}\right) .
$$

This corresponds to the $Z_{2}$ symmetry-breaking phase of the Ising model, which is also $\widetilde{Z}_{2}$ symmetric. We see that, indeed, the critical point of the Ising transition, plus its two neighboring gapped states, can be described by a gapless edge, and its neighbors, of $2+1 \mathrm{D} Z_{2}$ topological order.

When $J=B$, the boundary effective theory is gapless. From $H_{P}^{\mathrm{Is}}$ and $H_{A}^{\mathrm{Is}}$, we can obtain the gapless partition functions [31,50-52]:

$$
\left(\begin{array}{c}
Z_{1}(\tau, \bar{\tau}) \\
Z_{e}(\tau, \bar{\tau}) \\
Z_{m}(\tau, \bar{\tau}) \\
Z_{f}(\tau, \bar{\tau})
\end{array}\right)=\left(\begin{array}{c}
\left|\chi_{1}^{\mathrm{Is}} 1(\tau)\right|^{2}+\left|\chi_{\frac{1}{2}}^{\mathrm{IS}}(\tau)\right|^{2} \\
\left|\chi_{\frac{1}{\mathrm{Is}}}^{\mathrm{II}}(\tau)\right|^{2^{2}} \\
\left|\chi_{\frac{1}{16}}^{\mathrm{Is}}(\tau)\right|^{2} \\
\chi_{1}^{\mathrm{IS}} 1(\tau) \bar{\chi}_{\frac{1}{2}}^{\mathrm{Is}}(\tau)+\chi_{\frac{1}{2}}^{\mathrm{Is}}(\tau) \bar{\chi}_{1}^{\mathrm{I}}(\tau)
\end{array}\right),
$$

where $\chi_{i}^{\text {Is }}(\tau)$ are characters of Ising CFT, This corresponds to the critical point at the $Z_{2}$ symmetry-breaking transition.

Since $Z_{m}$ has the $Z_{2}$ symmetry twist, $Z_{m} \neq 0$ implies that the $Z_{2}$ symmetry is not spontaneously broken. Also $Z_{e}$ has the $\widetilde{Z}_{2}$ symmetry twist. Thus $Z_{e} \neq 0$ implies that the $\widetilde{Z}_{2}$ symmetry is not spontaneously broken. This is why we say the gapless critical point has full $Z_{2} \vee \widetilde{Z}_{2}$ categorical symmetry.

To see how the patch symmetry transformations for the $Z_{2} \vee \widetilde{Z}_{2}$ categorical symmetry act in the symmetric gapless point, we note that the patch symmetry operators have the following form at the symmetric gapless point:

$$
\begin{aligned}
& U_{Z_{2}}(i, j)=\widetilde{X}_{i-\frac{1}{2}} \widetilde{X}_{j+\frac{1}{2}} \sim \mu(i) \mu(j), \\
& U_{\widetilde{Z}_{2}}(i, j)=Z_{i} Z_{j} \sim \sigma(i) \sigma(j) .
\end{aligned}
$$

Here $\sigma$ and $\mu$ are two primary fields with scaling dimensions $\left(\frac{1}{16}, \frac{1}{16}\right)$ and $\left(\frac{1}{16}, \frac{1}{16}\right)$ of nonchiral Ising CFT. The operator product expansion with the fermion primary field $\psi$ in the Ising CFT is given by [4]

$$
\psi \sigma \sim \mu, \quad \bar{\psi} \mu \sim \sigma, \quad \sigma \mu \sim \psi+\bar{\psi} .
$$

The monodromy between $\mu$ and $\sigma$ is -1 . This reflects that $U_{Z_{2}}(i, j)$ and $U_{\widetilde{Z}_{2}}\left(i^{\prime}, j^{\prime}\right)$ have mutually $\pi$ statistics.

Therefore, the averages of the patch symmetry operators (i.e., the $Z_{2}$ and $\widetilde{Z}_{2}$ order parameters) have the form

$$
\begin{aligned}
& \left\langle U_{Z_{2}}(x, y)\right\rangle=\langle\mu(x) \mu(y)\rangle \sim|x-y|^{-1 / 4}, \\
& \left\langle U_{\widetilde{Z}_{2}}(x, y)\right\rangle=\langle\sigma(x) \sigma(y)\rangle \sim|x-y|^{-1 / 4} .
\end{aligned}
$$

They vanish when $|x-y| \rightarrow \infty$. Thus the Ising critical point has the full $Z_{2} \vee \widetilde{Z}_{2}$ categorical symmetry.

That in the modular invariant partition function in the Ising CFT there is only one excitation with scaling dimension $\left(\frac{1}{16}, \frac{1}{16}\right)$ is consistent with the fact that in this low-energy theory only either $U_{Z_{2}}(x, y)$ or $U_{\widetilde{Z}_{2}}(x, y)$ is the correlator of the local excitation, while the other is the patch symmetry operator acting on the local excitation.

The above partition functions are essentially determined by the $Z_{2} \vee \widetilde{Z}_{2}$ categorical symmetry. Remember that the $Z_{2} \vee \widetilde{Z}_{2}$ categorical symmetry is characterized by $2+1 \mathrm{D} Z_{2}$ topological order, which in turn is characterized by the following $S, T$ matrices:

$$
T^{Z_{2} \vee \widetilde{Z}_{2}}=\left(\begin{array}{cccc}
1 & 0 & 0 & 0 \\
0 & 1 & 0 & 0 \\
0 & 0 & 1 & 0 \\
0 & 0 & 0 & -1
\end{array}\right), S^{Z_{2} \vee \widetilde{Z}_{2}}=\left(\begin{array}{crrr}
\frac{1}{2} & \frac{1}{2} & \frac{1}{2} & \frac{1}{2} \\
\frac{1}{2} & \frac{1}{2} & -\frac{1}{2} & -\frac{1}{2} \\
\frac{1}{2} & -\frac{1}{2} & \frac{1}{2} & -\frac{1}{2} \\
\frac{1}{2} & -\frac{1}{2} & -\frac{1}{2} & \frac{1}{2}
\end{array}\right) .
$$

Through $S, T$ matrices, the categorical symmetry can determine the partition functions for low-energy fixed points via the following relation [31]:

$$
Z_{i}(\tau+1)=U_{i j}^{Z_{2} \vee \widetilde{Z}_{2}} Z_{j}(\tau), \quad Z_{i}(-1 / \tau)=S_{i j}^{Z_{2}} \widetilde{Z_{2}} Z_{j}(\tau) .
$$

For the gapped states in a system with $Z_{2} \vee \widetilde{Z}_{2}$ categorical symmetry, the partition functions $Z_{i}$ are $\tau$ independent positive integers with $Z_{1}=1$. We find that Eqs. (33) and (38) are the only two gapped solutions of Eq. (44). This confirms the result in Ref. [40]: gapped states must partially break categorical symmetry spontaneously.

Equation (39) is a gapless solution of Eq. (44) that has the full $Z_{2} \vee \widetilde{Z}_{2}$ categorical symmetry. Equation (44) also has other solutions with the full $Z_{2} \vee \widetilde{Z}_{2}$ categorical symmetry, such as

$$
\left(\begin{array}{c}
Z_{1} \\
Z_{e} \\
Z_{m} \\
Z_{f}
\end{array}\right)=\left(\begin{array}{c}
\left|\chi_{0}^{5,4}\right|^{2}+\left|\chi_{\frac{1}{10}}^{5,4}\right|^{2}+\left|\chi_{\frac{3}{5}}^{5,4}\right|^{2}+\left|\chi_{\frac{3}{2}}^{5,4}\right|^{2} \\
\left|\chi_{\frac{7}{16}}^{5}\right|^{2}+\left|\chi_{\frac{3}{80}}^{5,4}\right|^{2} \\
\left|\chi_{\frac{1}{5}, 4}^{5}\right|^{2}+\left|\chi_{\frac{3}{3}}^{5,4}\right|^{2} \\
\chi_{0}^{5,4} \bar{\chi}_{\frac{3}{2}}^{5,4}+\chi_{\frac{1}{10}}^{5,4^{16}} \bar{\chi}_{\frac{3}{5}}^{5,4}+\chi_{\frac{3}{5}}^{5,4} \bar{\chi}_{\frac{1}{10}}^{5,4}+\chi_{\frac{3}{2}}^{5,4} \bar{\chi}_{0}^{5,4}
\end{array}\right),
$$

where $\chi_{h}^{5,4}(\tau)$ are characters of the $(5,4)$ minimal model CFT (with central charge $c=\frac{7}{10}$ ).

We see that categorical symmetry can largely determine the gapless states where the categorical symmetry is not spontaneously broken, but not uniquely. However, the gapless 
TABLE I. The pointlike excitations and their fusion rules in 2+1D $S_{3}$ topological order. Here $b$ and $c$ correspond to pure $S_{3}$ flux excitations, $a^{1}$ and $a^{2}$ correspond to pure $S_{3}$ charge excitations, 1 corresponds to the trivial excitation, while $b^{1}, b^{2}$, and $c^{1}$ are charge-flux composites. $d, s$ are the quantum dimension and the topological spin of an excitation.

\begin{tabular}{lcccccccc}
\hline \hline$d, s$ & 1,0 & 1,0 & 2,0 & 2,0 & $2, \frac{1}{3}$ & $2,-\frac{1}{3}$ & 3,0 & $3, \frac{1}{2}$ \\
\hline$\otimes$ & $\mathbf{1}$ & $a^{1}$ & $a^{2}$ & $b$ & $b^{1}$ & $b^{2}$ & $c$ & $c^{1}$ \\
$\mathbf{1}$ & $\mathbf{1}$ & $a^{1}$ & $a^{2}$ & $b$ & $b^{1}$ & $b^{2}$ & $c^{2}$ & $c$ \\
$a^{1}$ & $a^{1}$ & $\mathbf{1}$ & $a^{2}$ & $b$ & $b^{1}$ & $b^{2}$ & $c$ & $c c^{1}$ \\
$a^{2}$ & $a^{2}$ & $a^{2}$ & $\mathbf{1} \oplus a^{1} \oplus a^{2}$ & $b^{1} \oplus b^{2}$ & $b \oplus b^{2}$ & $b \oplus b^{1}$ & $c \oplus c^{1}$ & $c c^{1}$ \\
$b$ & $b$ & $b$ & $b^{1} \oplus b^{2}$ & $\mathbf{1} \oplus a^{1} \oplus b$ & $b^{2} \oplus a^{2}$ & $b^{1} \oplus a^{2}$ & $c c^{1}$ & $c \oplus c^{1}$ \\
$b^{1}$ & $b^{1}$ & $b^{1}$ & $b \oplus b^{2}$ & $b^{2} \oplus a^{2}$ & $\mathbf{1} \oplus a^{1} \oplus b^{1}$ & $b \oplus a^{2}$ & $c \oplus c^{1}$ & $c^{1}$ \\
$b^{2}$ & $b^{2}$ & $b^{2}$ & $b \oplus b^{1}$ & $b^{1} \oplus a^{2}$ & $b \oplus a^{2}$ & $\mathbf{1} \oplus a^{1} \oplus b^{2}$ & $\mathbf{1} \oplus a^{2} \oplus b \oplus b^{1} \oplus b^{2}$ & $a^{1} \oplus a^{2} \oplus b \oplus b^{1} \oplus b^{2}$ \\
$c$ & $c$ & $c^{1}$ & $c \oplus c^{1}$ & $c \oplus c^{1}$ & $c \oplus c^{1}$ & $c \oplus c^{1}$ & $c \oplus c^{1} \oplus a^{2} \oplus b \oplus b^{1} \oplus b^{2}$ & $\mathbf{1} \oplus a^{2} \oplus b \oplus b^{1} \oplus b^{2}$ \\
$c^{1}$ & $c^{1}$ & $c$ & $c \oplus c^{1}$ & $c \oplus c^{1}$ & $c \oplus c^{1}$ & $c \oplus c^{1}$ & $a^{1}$ \\
\hline \hline
\end{tabular}

states with larger heat capacity may have additional emergent categorical symmetry. Therefore, we consider minimal gapless states (i.e., with minimal central charge $c$ ) with the full categorical symmetry. For $Z_{2} \vee \widetilde{Z}_{2}$ categorical symmetry, there is only one minimal gapless state Eq. (39). For categorical symmetry characterized by $2+1 \mathrm{D} S_{3}=Z_{3} \rtimes Z_{2}$ gauge theory there is also only one minimal gapless state (see Table I) [31]:

$$
\begin{aligned}
& Z_{11}=\left|\chi_{0}^{6,5}\right|^{2}+\left|\chi_{3}^{6,5}\right|^{2}+\left|\chi_{\frac{2}{5}}^{6,5}\right|^{2}+\left|\chi_{\frac{7}{5}}^{6,5}\right|^{2}, \\
& Z_{a^{1}}=\chi_{0}^{6,5} \bar{\chi}_{3}^{6,5}+\chi_{3}^{6,5} \bar{\chi}_{0}^{6,5}+\chi_{\frac{2}{5}}^{6,5} \bar{\chi}_{\frac{7}{5}}^{6,5}+\chi_{\frac{7}{5}}^{6,5} \bar{\chi}_{\frac{2}{5}}^{6,5}, \\
& Z_{a^{2}}=\left|\chi_{\frac{2}{3}}^{6,5}\right|^{2}+\left|\chi_{\frac{1}{15}}^{6,5}\right|^{2}, \\
& Z_{b}=\left|\chi_{\frac{2}{3}}^{6,5}\right|^{2}+\left|\chi_{\frac{1}{15}}^{6,5}\right|^{2}, \\
& Z_{b^{1}}=\chi_{0}^{6,5} \bar{\chi}_{\frac{2}{3}}^{6,5}+\chi_{3}^{6,5} \bar{\chi}_{\frac{2}{3}}^{6,5}+\chi_{\frac{2}{5}}^{6,5} \bar{\chi}_{\frac{1}{15}}^{6,5}+\chi_{\frac{7}{5}}^{6,5} \bar{\chi}_{\frac{1}{15}}^{6,5}, \\
& Z_{b^{2}}=\chi_{\frac{2}{3}}^{6,5} \bar{\chi}_{0}^{6,5}+\chi_{\frac{2}{3}}^{6,5} \bar{\chi}_{3}^{6,5}+\chi_{\frac{1}{15}}^{6,5} \bar{\chi}_{\frac{2}{5}}^{6,5}+\chi_{\frac{1}{15}}^{6,5} \bar{\chi}_{\frac{7}{5}}^{6,5}, \\
& Z_{c}=\left|\chi_{\frac{1}{8}}^{6,5}\right|^{2}+\left|\chi_{\frac{13}{8}}^{6,5}\right|^{2}+\left|\chi_{\frac{1}{40}}^{6,5}\right|^{2}+\left|\chi_{\frac{21}{40}}^{6,5}\right|^{2}, \\
& Z_{c^{1}}=\chi_{\frac{1}{8}}^{6,5} \bar{\chi}_{\frac{13}{8}}^{6,5}+\chi_{\frac{13}{8}}^{6,5} \bar{\chi}_{\frac{1}{8}}^{6,5}+\chi_{\frac{1}{40}}^{6,5} \bar{\chi}_{\frac{21}{40}}^{6,5}+\chi_{\frac{21}{40}}^{6,5} \bar{\chi}_{\frac{1}{40}}^{6,5},
\end{aligned}
$$

where $\chi_{h}^{6,5}(\tau)$ are characters of the $(6,5)$ minimal model (with central charge $c=\frac{4}{5}$ ).

The above examples strongly suggest that categorical symmetry characterized by $2+1 \mathrm{D} Z_{2}$ topological order allows us to determine one or a few minimal gapless states via Eq. (44). This points to a direction that gapless states are largely (possibly even uniquely) determined by categorical symmetries, i.e., by topological order in one higher dimension.

\section{III. $Z_{2}$ SYMMETRY AND $Z_{2}^{(1)}$ 1-SYMMETRY IN THE 2 + 1D ISING MODEL}

In two dimensions, it is well known that the critical point for the $Z_{2}$ symmetry-breaking transition has the $Z_{2}$ symmetry. In this section, we show that the critical point also has a one symmetry.

Let us consider the following two models: the $Z_{2}$ Ising model and $Z_{2}$ gauge model on the square lattice. We will demonstrate that the $Z_{2}$ Ising model restricted to the $Z_{2}$ even (chargeless) sector is exactly dual to the lattice $Z_{2}$-link model in the limit where the $Z_{2}$ vortex has an infinity gap.

The $Z_{2}$ Ising model is given by

$$
H=-J \sum_{l} Z_{i_{1}(l)} Z_{i_{2}(l)}-B \sum_{i} X_{i}
$$

where $\sum_{l}$ sums over all links, $\sum_{i}$ sums over all vertices, and $i_{1}(l)$ and $i_{2}(l)$ are two vertices connected by the link $l$. The lattice $Z_{2}$ gauge model is given by

$$
\widetilde{H}=-J \sum_{l} \widetilde{Z}_{l}-B \sum_{i} \prod_{l \supset i} \widetilde{X}_{l}+U \sum_{s}\left(1-\prod_{l \in S} \widetilde{Z}_{l}\right),
$$

where $\sum_{i}$ sums over all sites, $\sum_{s}$ sums over all squares, $\prod_{l \supset i}$ is a product over all the four links that contain vertex $i$, and $\prod_{l \in s}$ is a product over all the four links on the boundary of square $s$. We consider the limit $U \rightarrow+\infty$.

The two models can be mapped into each other via the map that preserves the operator algebra:

$$
X_{i} \rightarrow \prod_{l \supset i} \tilde{X}_{l}, \quad Z_{i_{1}(l)} Z_{i_{2}(l)} \rightarrow \widetilde{Z}_{l} .
$$

We will refer to such a map as the "duality" map, or "gauging" in a looser sense, from a pure matter theory to a pure gauge theory.

The Ising model $H$ has a global $Z_{2}$ symmetry generated by

$$
U_{Z_{2}}=\prod_{i} X_{i}
$$

After duality, it is mapped into an identity operator 1 . The lattice $Z_{2}$ gauge model has a $Z_{2}^{(1)} 1$-symmetry generated by the Wilson-line operators along any closed path $C$ :

$$
U_{Z_{2}^{(1)}}(C)=\prod_{l \in C} \widetilde{Z}_{l}
$$

It corresponds to an identity operator in the Ising model under the duality map.

Next we compare the low-energy sub-Hilbert space of the two models. Assume the space to be a torus with $N=L \times L$ vertices. The Hilbert space of the Ising model has a dimension $2^{N}$. The subspace of $Z_{2}$-symmetric states, $\mathcal{V}_{\text {Ising }}^{\text {symm }}$, has a dimension $2^{N-1}$. The Hilbert space of the $Z_{2}$ gauge model has a dimension $2^{2 N}$. In the $U \rightarrow+\infty$ limit, the low-energy 
subspace has a dimension $2^{N} \times 2$, The extra factor 2 is due to the operator identity $\prod_{s} \prod_{l \in \mathcal{S}} \widetilde{Z}_{l}=1$. In the low-energy sub-Hilbert space, we have $\prod_{l \in s} \widetilde{Z}_{l}=1$ and

$$
U_{Z_{2}^{(1)}}(C)=U_{Z_{2}^{(1)}}\left(C^{\prime}\right)
$$

if $C$ can be deformed into $C^{\prime}$. Now we consider the subspace, $\mathcal{V}_{\text {gauge }}^{\text {symm }}$, of the low-energy Hilbert space where $U_{Z_{2}^{(1)}}\left(S_{x}^{1}\right)=$ $U_{Z_{2}^{(1)}}\left(S_{y}^{1}\right)=1$, where $S_{x}^{1}$ and $S_{y}^{1}$ are the two noncontractible loops wrapping around the system in $x$ and $y$ directions. $\mathcal{V}_{\text {gauge }}^{\text {symm }}$ has a dimension $2^{N-1} . H$ in $\mathcal{V}_{\text {Ising }}^{\text {symm }}$ and $\widetilde{H}$ in $\mathcal{V}_{\text {gauge }}^{\text {symm }}$ equivalent via a unitary transformation. In this sense, the Ising model Eq. (47) is exactly dual to the $Z_{2}$ gauge model Eq. (48).

Now let us consider the ground states. Let us assume $J, B>0$. It is interesting to note that, for $B \gg J$, the trivial phase of the Ising model is mapped to the topologically ordered phase (the $Z_{2}^{(1)}$ symmetry-breaking phase) of the $Z_{2}$ gauge model, while for $B \ll J$ the $Z_{2}$ symmetry-breaking phase of the Ising model is mapped to the trivial phase (the symmetric phase of the $Z_{2}^{(1)}$ one symmetry) of the $Z_{2}$ gauge model. At the gapless critical point of the $2+1 \mathrm{D} Z_{2}$ symmetry-breaking transition (also the $Z_{2}^{(1)}$ one symmetrybreaking transition), we have the $Z_{2} \vee Z_{2}^{(1)}$ symmetry which is not spontaneously broken. Therefore, we show the appearance of 1 -symmetry of the ground state at the $2+1 \mathrm{D} Z_{2}$ symmetrybreaking transition.

Now we discuss the charges of the categorical symmetry $Z_{2} \vee Z_{2}^{(1)}$. We will find that in the sub-Hilbert space we can only create charges the total of which is neutral, as measured by the global symmetry operators, while the charge in a finite region is not neutral and can be measured by patch operators. We may call this kind of charge excitations neutral charges.

A single charge of $Z_{2}$ symmetry is a $e$ particle on a site $\left[Z_{i}=-1\right.$ in the Ising model Eq. (47)]. The conserved charge of $Z_{2}$ symmetry, however, is two $e$ particles, living on two sites, or rather $S^{0}$. The charge of the $Z_{2}^{(1)}$ symmetry is an open $Z_{2}$ vortex string, living on $D^{1}$. The conserved charge of the $\widetilde{Z}_{2}{ }^{(1)}$ symmetry is a closed contractible $Z_{2}$ vortex loop living on $S^{1}$; let us call it a $s$ string. In the sub-Hilbert space, we can only create conserved charges, excitation on $S^{0}$ and $S^{1}$. The operator for a pair of $e$ particles at site $i$ and site $j$ is $Z_{i} Z_{j}$. In the $Z_{2}$ gauge theory, the operator is dual to $U_{Z_{2}^{(1)}}\left(C_{i j}\right)=$ $\prod_{l \in C_{i j}} \widetilde{Z}_{l}$, where $C_{i j}$ is any path from $i$ site to $j$ site. It is also the patch (or part) of the generators of the $Z_{2} 1$ symmetry.

A $s$ string is created by

$$
U_{Z_{2}}\left[\left(S^{1}\right)^{\vee}\right]=\prod_{l \in\left(S^{1}\right)^{\vee}} \tilde{X}_{l},
$$

where $\left(S^{1}\right)^{\vee}$ is a contractible loop on the dual lattice. It corresponds to create $\widetilde{Z}_{l}=-1$ along the loop in the $Z_{2}$ gauge model Eq. (48). In the Ising model, the $s$ string operator is dualed from the patch operator of the $Z_{2}$ global symmetry,

$$
U_{Z_{2}}\left(C^{\vee}\right)=\prod_{i \in D^{2}} X_{i},
$$

where $D^{2}$ is the disk the boundary of which is $C^{\vee}$. This charge, when measured by $Z_{2}^{(1)}$ symmetry generator Eq. (51), is neutral. Yet, when measured by part of the generator $U_{Z_{2}}\left(C_{i j}\right)$, it has charge -1 when there are $C^{\vee}$ circles around a single end point of $C_{i j}$, either $i$ or $j$.

That is to say, the two kinds of patch operators satisfy the following relation:

$$
U_{Z_{2}^{(1)}}\left(C_{i j}\right) U_{Z_{2}}\left(C^{\vee}\right)=-U_{Z_{2}}\left(C^{\vee}\right) U_{Z_{2}^{(1)}}\left(C_{i j}\right),
$$

when only one $e$ particle on either $i$ or $j$ site is circled by the loop excitation along $C^{\vee}$. This reveals the mutual $\pi$ statistics between the $e$ and $s$ excitation.

In summary, in the sub-Hilbert space, there are states with both conserved $e$ charges and a conserved $s$ string, either the Ising model description or the $Z_{2}$ gauge theory description. They are created by patch operators. And one kind of patch operator can measure the existence of the other. In other words, the charge of $Z_{2}$ and that of $Z_{2}^{(1)}$ have mutual statistics. The $Z_{2} \vee Z_{2}^{(1)}$ symmetry (or more precisely, the $Z_{2} \vee Z_{2}^{(1)}$ categorical symmetry) is the conservation of $e$ particles and $s$ strings, together with the mutual statistics.

The above theory with sub-Hilbert space also describes the boundary of the $3+1 \mathrm{D} Z_{2}$ gauge theory with $Z_{2}$ charge $e$ and $Z_{2}$-vortex string $s$, where $e$ particles and $s$ strings have low energies only at the boundary. In $3+1 \mathrm{D} Z_{2}$ topological gauge theory, the $e$ excitations (living on $S^{0}$, composed of two points) and $s$ string [living on $\left(S^{1}\right)^{\vee}$ ] are created by the following operators defined on the open string $C$ on the lattice and a contractible membrane $\left(D^{2}\right)^{\vee}$ on the dual lattice:

$$
\begin{gathered}
U_{Z_{2}}(C)=\prod_{l \in C} \widetilde{Z}_{l}, \\
U_{Z_{2}^{(2)}}\left[\left(D^{2}\right)^{\vee}\right]=\prod_{l \in\left(D^{2}\right)^{\vee}} \widetilde{X}_{l} .
\end{gathered}
$$

The $s$ vortex loop on the boundary of $U_{Z_{2}^{(2)}}$ brought to the boundary of the $3 \mathrm{D}$ spatial lattice is the neutral $Z_{2}^{(1)}$ charge in the $2 d$ Ising model Eq. (47) or $Z_{2}$-link model Eq. (48) with a sub-Hilbert space, respectively. It is neutral in the sense that the membrane Eq. (57) creating it commutes with any $U_{Z_{2}}\left(S^{1}\right)$ on a closed string $S^{1}$.

Furthermore, the $3+1 \mathrm{D}$ bulk has a highly nontrivial effect on the boundary: in a gapped ground state of the $2+1 \mathrm{D}$ model (47), one and only one of the $Z_{2}$ and $Z_{2}^{(1)}$ symmetries must be spontaneously broken. Pictorially speaking, this is because the two topological excitations with mutual statistics cannot condense simultaneously. Thus, a ground state of model (47) with the full $Z_{2} \vee Z_{2}^{(1)}$ categorical symmetry must be gapless. This is a consequence of a $2+1 \mathrm{D}$ noninvertible gravitational anomaly [31] and the $Z_{2} \vee Z_{2}^{(1)}$ categorical symmetry characterized by $3+1 \mathrm{D} Z_{2}$ topological order (i.e., $Z_{2}$ gauge theory).

\section{MODEL WITH ANOMALOUS SYMMETRY AS THE BOUNDARY OF TOPOLOGICAL ORDER IN ONE HIGHER DIMENSION}

Through the examples above, we have shown that a model with a finite symmetry $G$, when restricted in the symmetric sector, can be viewed as the boundary of $G$-gauge theory in one higher dimension. The conservation (the fusion rules) of the pointlike gauge charge and codimension-2 gauge flux give rise to the symmetry and algebraic higher symmetry 
(the combination of which becomes the so-called categorical symmetry) of the $G$-symmetric model. The categorical symmetry is not spontaneously broken at the critical point of the symmetry-breaking transition (see Sec. V for more details).

We know that a $G$-gauge theory can be twisted and becomes Dijkgraaf-Witten theory [53]. We will show that the boundary of such twisted $G$-gauge theory has an anomalous $G$ symmetry. This implies that a system with anomalous $G$ symmetry [54,55] also has an algebraic higher symmetry. The combination of the two symmetries corresponds to the categorical symmetry described by the twisted $G$-gauge theory in one higher dimension. Such a system has a gapless state, where the categorical symmetry is not spontaneously broken. Also, a state with the unbroken categorical symmetry must be gapless. And the gapped states of the system must spontaneously break the anomalous $G$ symmetry.

\section{A. Boundary of the double-semion model}

In this section, we will study the boundary of the doublesemion (DS) model (i.e., the twisted $Z_{2}$ gauge theory in the $2+1 \mathrm{D}$ case) to illustrate the above result.

A $2+1 \mathrm{D}$ DS topological order has four types of excitations $11, s, s^{*}$, and $b$. Here $s, s^{*}$, and $f$ are topological excitations; $s$ and $s^{*}$ are semions with statistics $\pm i$; and $b$ is a boson. They satisfy the following fusion relation:

$$
s \otimes s=11, s^{*} \otimes s^{*}=11, \quad b \otimes b=11, \quad s \otimes s^{*}=b .
$$

$s$ and $s$ have a mutual $\pi$ statistics and $s$ and $s^{*}$ have a mutual boson statistics. As a result, $s$ and $b$ have a mutual $\pi$ statistics.

We consider a gapped boundary from condensing $b$ excitations. Since $b \otimes b=11$ and $b$ particles have mod- 2 conservation, we assume the $b$ condensation gives rise to two degenerate ground states, one with $Z_{i}=1$ and the other with $Z_{i}=-1$. The domain wall between $Z_{i}=1$ and -1 regions corresponds a $s$ particle.

We would like to point out that, on the boundary, although the $s$-type particle and $e$-type particle (in the $Z_{2}$ gauge theory discussed before) have the same fusion rule $s \otimes s=11$ and $e \otimes e=1$, their fusion $F$ tensors are different [56,57]. In particular, fusings of three $s$-type particles into one $s$-type particle in two different ways differ by a phase -1 :

$$
[s s s \rightarrow \mathbb{1} \mathbb{1} s \rightarrow \mathbb{1} s \mathbb{1}]=(-)[s s s \rightarrow s \mathbb{1} \rightarrow \mathbb{1} s \mathbb{1}] .
$$

In contrast, fusings of three $e$-type particles (described by $X_{i}=-1$ ) into one $e$-type particle in two different ways have the same phase:

$$
[e e e \rightarrow \mathbb{1} 11 e \rightarrow \mathbb{1 l} e \mathbb{1 1}]=[e e e \rightarrow e \mathbb{1 1} \rightarrow \mathbb{1 l} e \mathbb{1 1}]
$$

For the boundary of $Z_{2}$ topological order, the above two processes of fusing $e$ particles are induced, respectively, by a pair-annihilation operator $X_{i}^{+} X_{i+1}^{+}$and a hopping operator $X_{i}^{+} X_{i+1}^{-}+X_{i}^{-} X_{i+1}^{+}$, where

$$
X^{ \pm}=\frac{1}{2}(Y \pm i Z)
$$

Indeed, we have

$$
\begin{aligned}
& \left(X_{i-1}^{+} X_{i}^{-}+X_{i-1}^{-} X_{i}^{+}\right)\left(X_{i}^{+} X_{i+1}^{+}\right)|e e e\rangle \\
& \quad=\left(X_{i}^{+} X_{i+1}^{-}+X_{i}^{-} X_{i+1}^{+}\right)\left(X_{i-1}^{+} X_{i}^{+}\right)|e e e\rangle .
\end{aligned}
$$

The pair-annihilation operator $Z_{i}^{+} Z_{i+1}^{+}$and hopping operator $Z_{i}^{+} Z_{i+1}^{-}+Z_{i}^{-} Z_{i+1}^{+}$are allowed local operations, and we can use them to construct effective boundary Hamiltonian

$$
\begin{aligned}
H_{P}^{\mathrm{Is}}= & -\sum_{i=1}^{L} J_{1}\left(Z_{i}^{+} Z_{i+1}^{-}+Z_{i}^{-} Z_{i+1}^{+}\right)+J_{2}\left(Z_{i}^{+} Z_{i+1}^{+}+\text {H.c. }\right) \\
& -B \sum_{i=1}^{L} Z_{i}
\end{aligned}
$$

which describes the boundary of $2+1 \mathrm{D} Z_{2}$ topological order.

For the boundary of DS topological order, the two processes for fusing $s$ particles Eq. (59) are also induced by a pair-annihilation operator and a hopping operator. Here we choose the hopping operator to be $X_{i}-Z_{i-1} X_{i} Z_{i+1}$, which shifts a domain wall from $i-\frac{1}{2}$ to $i+\frac{1}{2}$, or $i+\frac{1}{2}$ to $i-\frac{1}{2}$. The pair-annihilation or pair-creation operator is given by $Z_{i-1}\left(X_{i}+Z_{i-1} X_{i} Z_{i+1}\right)$, which creates or annihilates a pair of domain walls at $i+\frac{1}{2}$ and $i-\frac{1}{2}$.

For three $s$-type particles (the domain walls) at $i-\frac{1}{2}, i+$ $\frac{1}{2}, i+\frac{3}{2}$, we indeed have

$$
\begin{aligned}
- & \left(X_{i+1}-Z_{i} X_{i+1} Z_{i+2}\right) Z_{i-1}\left(X_{i}+Z_{i-1} X_{i} Z_{i+1}\right)|s s s\rangle \\
& =\left(X_{i}-Z_{i-1} X_{i} Z_{i+1}\right) Z_{i}\left(X_{i+1}+Z_{i} X_{i+1} Z_{i+2}\right)|s s s\rangle,
\end{aligned}
$$

where $|s s s\rangle=\left|\uparrow_{i-1} \downarrow_{i} \uparrow_{i+1} \downarrow_{i+2}\right\rangle$.

Now we can construct the boundary effective theory for the $b$ condensed boundary of DS topological order. We note that such a boundary contains a gapped excitation that corresponds to the $s$-type particle. One might expect a second boundary excitation corresponding to the $s^{*}$-type particle. However, since $b$ is condensed on the boundary, the $s$-type particle and the $s^{*}$-type particle are actually equivalent on the boundary. The simplest boundary effective lattice Hamiltonian that describes the gapped $s$ particles has the form

$$
H=-B \sum_{i} Z_{i} Z_{i+1}, \quad B>0,
$$

which has two degenerate ground states and the $s$ particles correspond to domain walls.

Using the above allowed local operations $X_{i}-Z_{i-1} X_{i} Z_{i+1}$ and $Z_{i-1}\left(X_{i}+Z_{i-1} X_{i} Z_{i+1}\right)$, we can construct a more general boundary effective theory:

$$
\begin{aligned}
H^{\mathrm{DS}}= & -B \sum_{i=1}^{L} Z_{i} Z_{i+1}-J_{1} \sum_{i=1}^{L}\left(X_{i}-Z_{i-1} X_{i} Z_{i+1}\right) \\
& +J_{2} \sum_{i=1}^{L} Z_{i-1}\left(X_{i}+Z_{i-1} X_{i} Z_{i+1}\right)
\end{aligned}
$$

where site $i$ and site $(i+L)$ are identified.

We note that the above Hamiltonian is not invariant under the spin-flip transformation $\prod_{i} X_{i}$. In fact, it is invariant under a non-on-site transformation [58]:

$$
U_{Z_{2}}=\prod_{i} X_{i} \prod_{i} s_{i, i+1},
$$


where $s_{i j}$ acts on two spins as

$$
\begin{aligned}
s_{i j}= & |\uparrow \uparrow\rangle\langle\uparrow \uparrow|+| \downarrow \uparrow\rangle\langle\downarrow \uparrow|-| \uparrow \downarrow\rangle \\
& \times\langle\uparrow \downarrow|+| \downarrow \downarrow\rangle\langle\downarrow \downarrow| \\
= & \frac{1}{2}\left(1-Z_{i}+Z_{j}+Z_{i} Z_{j}\right) .
\end{aligned}
$$

The transformation has a simple picture: it flips all the spins and includes a $(-)^{N_{\uparrow \rightarrow \downarrow}}$ phase, where $N_{\uparrow \rightarrow \downarrow}$ is the number of the $\uparrow \rightarrow \downarrow$ domain wall. We see that the transformation is a $Z_{2}$ transformation (i.e., square to 1 ). From Appendix $C$, the $Z_{2}$ transformation has the following action:

$$
\begin{aligned}
& Z_{i} \leftrightarrow-Z_{i}, \\
& X_{i} \leftrightarrow-Z_{i-1} X_{i} Z_{i+1} .
\end{aligned}
$$

We find that Eq. (66) is invariant under the $Z_{2}$ transformation.

From the above discussion, we see that the different fusion properties lead to different local operators. The boundary effective theories for $Z_{2}$ topological order and for the double semion topological order are different. In particular, the boundary effective theory for $Z_{2}$ topological order has an on-site $Z_{2}$ symmetry, while the boundary effective theory for the double semion topological order has a non-on-site $Z_{2}$ symmetry. The non-on-site $Z_{2}$ symmetry $U_{Z_{2}}$ implies that the model (66) cannot have a gapped $Z_{2}$-symmetric ground state [58].

\section{B. $\tilde{Z}_{2}$ dual symmetry}

We have seen that a $1+1 \mathrm{D}$ lattice model (66) with an anomalous $Z_{2}$ symmetry (non-on-site symmetry $[55,58]$ ) can be viewed as a boundary of twisted $2+1 \mathrm{D} Z_{2}$ gauge theory (i.e., DS topological order). The anomalous $Z_{2}$ symmetry comes from the mod- 2 conserved $b$ particles. The mod-2 conserved $s$ particles will give rise to another symmetry, which will be referred to as dual $\widetilde{Z}_{2}$ symmetry. In other words, we claim that the model (66) has both the $Z_{2}$ symmetry and the $\widetilde{Z}_{2}$ symmetry.

To see the $\widetilde{Z}_{2}$ symmetry explicitly, we do a dual transformation on the model (66):

$$
\begin{aligned}
Z_{i} Z_{i+1} & \rightarrow \widetilde{Z}_{i+\frac{1}{2}} \\
X_{i} & \rightarrow \widetilde{X}_{i-\frac{1}{2}} \widetilde{X}_{i+\frac{1}{2}} \\
Z_{i} & \rightarrow \prod_{j \leqslant i} \widetilde{Z}_{j-\frac{1}{2}}
\end{aligned}
$$

We find

$$
\begin{aligned}
X_{i} & -Z_{i-1} X_{i} Z_{i+1}=X_{i}+Z_{i-1} Z_{i} X_{i} Z_{i} Z_{i+1} \\
& \rightarrow \widetilde{X}_{i-\frac{1}{2}} \widetilde{X}_{i+\frac{1}{2}}+\widetilde{Z}_{i-\frac{1}{2}} \widetilde{X}_{i-\frac{1}{2}} \widetilde{X}_{i+\frac{1}{2}} \widetilde{Z}_{i+\frac{1}{2}} \\
& =\widetilde{X}_{i-\frac{1}{2}} \widetilde{X}_{i+\frac{1}{2}}+\widetilde{Y}_{i-\frac{1}{2}} \widetilde{Y}_{i+\frac{1}{2}}, \\
X_{i} & +Z_{i-1} X_{i} Z_{i+1}=X_{i}-Z_{i-1} Z_{i} X_{i} Z_{i} Z_{i+1} \\
& \rightarrow \widetilde{X}_{i-\frac{1}{2}} \widetilde{X}_{i+\frac{1}{2}}-\widetilde{Z}_{i-\frac{1}{2}} \widetilde{X}_{i-\frac{1}{2}} \widetilde{X}_{i+\frac{1}{2}} \widetilde{Z}_{i+\frac{1}{2}} \\
& =\widetilde{X}_{i-\frac{1}{2}} \widetilde{X}_{i+\frac{1}{2}}-\widetilde{Y}_{i-\frac{1}{2}} \widetilde{Y}_{i+\frac{1}{2}} \cdot
\end{aligned}
$$

The duality transformation changes the Hamiltonian (66) into

$$
\begin{aligned}
\widetilde{H}^{\mathrm{DS}}= & +J_{2} \sum_{i} \prod_{j<i} \widetilde{Z}_{j-\frac{1}{2}}\left(\widetilde{X}_{i-\frac{1}{2}} \widetilde{X}_{i+\frac{1}{2}}-\widetilde{Y}_{i-\frac{1}{2}} \tilde{Y}_{i+\frac{1}{2}}\right) \\
& -B \sum_{i} \widetilde{Z}_{i+\frac{1}{2}}-J_{1} \sum_{i}\left(\widetilde{X}_{i-\frac{1}{2}} \widetilde{X}_{i+\frac{1}{2}}+\widetilde{Y}_{i-\frac{1}{2}} \widetilde{Y}_{i+\frac{1}{2}}\right) .
\end{aligned}
$$

We see that the dual $\widetilde{Z}_{2}$ symmetry is generated by

$$
U_{\widetilde{Z}_{2}}=\prod_{i} \widetilde{Z}_{i+\frac{1}{2}} .
$$

This way, we obtain the explicit expression of the dual $\widetilde{Z}_{2}$ symmetry. The on-site $\widetilde{Z}_{2}$ symmetry $U_{\widetilde{Z}_{2}}$ implies that the model (66) can have a gapped $\widetilde{Z}_{2}$-symmetric ground state, which corresponds to a $Z_{2}$ symmetry-breaking state.

In the dual model, $\widetilde{Z}_{i+\frac{1}{2}}=1$ describes a site with no semion $s$, while $\widetilde{Z}_{i+\frac{1}{2}}=-1$ describes a site occupied with a semion $s$. The term $\widetilde{X}_{i-\frac{1}{2}} \tilde{X}_{i+\frac{1}{2}}+\widetilde{Y}_{i-\frac{1}{2}} \tilde{Y}_{i+\frac{1}{2}}$ is the hopping term for the $s$ particle, while the term $\prod_{j<i}^{2} \widetilde{Z}_{j-\frac{1}{2}}\left(\widetilde{X}_{i-\frac{1}{2}} \tilde{X}_{i+\frac{1}{2}}-\widetilde{Y}_{i-\frac{1}{2}} \tilde{Y}_{i+\frac{1}{2}}\right)$ creates a pair of $s$ particles.

\section{APPEARANCE OF ALGEBRAIC HIGHER SYMMETRY AT THE SYMMETRY-BREAKING TRANSITION FOR GENERAL FINITE SYMMETRY}

In the previous section, we show the categorical symmetry in $1+1 \mathrm{D}$ and $2+1 \mathrm{D}$ models with local degrees of freedom taking values in $Z_{2}$. In this section, we generalize the discussion to any $(n+1) \mathrm{D}$ lattice models with local degrees of freedom taking values in any finite group $G$. As above, we discuss the lattice model in terms of two descriptions, generalizing the Ising model and the $Z_{2}$-link model to the $G$-matter model and the $G$-link model. A major distinction is that when $G$ is non-Abelian the 0 -symmetry in the $G$-link model is a global symmetry that is not reduced to specifying boundary conditions. We will show the emergence of categorical symmetry at and off the critical point of Landau symmetry-breaking transition in these models.

\section{A. Duality point of view}

We consider two lattice models defined on the triangulation of $n$-dimensional space. The vertices of the triangulation are labeled by $i$, the links are labeled by $i j$, etc.

In the first model, which we may call the $G$-matter model, the physical degrees of freedom live on the vertices and are labeled by group elements $g$ of a finite group $G$. The manybody Hilbert space is spanned in the following local basis:

$$
\left|\left\{g_{i}\right\}\right\rangle, \quad g_{i} \in G .
$$

The Hamiltonian is given by

$$
H_{1}=-J \sum_{i j} \delta\left(g_{i} g_{j}^{-1}\right)-B \sum_{i} \sum_{h \in G} L_{h}(i),
$$

where

$$
\delta(g)= \begin{cases}1, & \text { if } g=1 \\ 0, & \text { otherwise }\end{cases}
$$


Also, the operator $L_{h}(i)$ is given by

$$
L_{h}(i)\left|g_{1}, \cdots, g_{i}, \cdots, g_{N}\right\rangle=\left|g_{1}, \cdots, h g_{i}, \cdots, g_{N}\right\rangle .
$$

The Hamiltonian $H_{1}$ has an on-site $G$ zero symmetry:

$$
U_{h} H_{1}=H_{1} U_{h}, \quad U_{h}=\prod_{i} L_{h}(i)
$$

We see that, when $J \gg B, H_{1}$ is in the symmetry-breaking phase, and, when $J \ll B, H_{1}$ is in the symmetric phase.

Our second bosonic lattice model, which we may call the $G$-link model, has degrees of freedom living on the links. On an oriented link $i j$ pointing from $i$ site to $j$ site, the degrees of freedom are labeled by $g_{i j} \in G$. The many-body Hilbert space has the following local basis:

$$
\left|\left\{g_{i j}\right\}\right\rangle, \quad g_{i j} \in G .
$$

Here, $g_{i j}$ 's on links with opposite orientations satisfy

$$
g_{i j}=g_{j i}^{-1} .
$$

The second model is related to the first model. A state $\left|g_{1}, \cdots, g_{i}, \cdots, g_{N}\right\rangle$ in the first model is mapped to a state $\left|\cdots, g_{i j}, \cdots\right\rangle$ in the second model where $g_{i j}=g_{i} g_{j}^{-1}$.

This connection allows us to design the Hamiltonian of the second model as

$$
\begin{aligned}
H_{2}= & -J \sum_{i j} \delta\left(g_{i j}\right)-B \sum_{i} \sum_{h \in G} Q_{h}(i) \\
& -U \sum_{i j k} \delta\left(g_{i j} g_{j k} g_{i k}^{-1}\right),
\end{aligned}
$$

where the star term $Q_{h}(i)$ acts on all the links that connect to the vertex $i$ :

$$
\begin{aligned}
& Q_{h}(i)\left|\cdots, g_{i j}, g_{k i}, g_{j k}, \cdots\right\rangle \\
& \quad=\left|\cdots, h g_{i j}, g_{k i} h^{-1}, g_{j k}, \cdots\right\rangle,
\end{aligned}
$$

and the plaquette term acts as a projection to zero-flux configurations. The second model has an algebraic $(n-1)$ symmetry, denoted as $G^{(n-1)}[8]$,

$$
W_{q}\left(S^{1}\right) H_{2}=H_{2} W_{q}\left(S^{1}\right), \quad W_{q}\left(S^{1}\right)=\operatorname{Tr} \prod_{i j \in S^{1}} R_{q}\left(g_{i j}\right),
$$

for any loop $S^{1}$ formed by links, where $R_{q}$ is an irreducible representation of $G$. We see that the algebraic $(n-1)$ symmetry $G^{(n-1)}$ is generated by the Wilson loop operators $W_{q}\left(S^{1}\right)$, for all loops $S^{1}$ and all irreducible representations $q$. We note that the algebraic 0 -symmetry $G^{(0)}$ is different from the usual zero symmetry characterized by a group $G$, when $G$ is non-Abelian. But when $G$ is Abelian the algebraic zero symmetry $G^{(0)}$ happens to be the usual zero symmetry $G$. Also, for Abelian $G, G^{(n)}$ is a $n$-symmetry described by a higher group. But, for non-Abelian $G, G^{(n)}$ is an algebraic $n$-symmetry beyond a higher group.

The Hamiltonian $\mathrm{H}_{2}$ has the algebraic $(n-1)$-symmetry, because the $Q_{h}(i)$ term in the Hamiltonian can be viewed as a "gauge" transformation and the Wilson loop operator $W_{q}\left(S^{1}\right)$ is gauge invariant, and hence

$$
W_{q}\left(S^{1}\right) Q_{h}(i)=Q_{h}(i) W_{q}\left(S^{1}\right) .
$$

$W_{q}\left(S^{1}\right)$ commutes with other terms in $H_{2}$ since they are all diagonal in the $\left|\left\{g_{i j}\right\}\right\rangle$ basis.

In the limit $|B| \ll J \ll U$, the ground state of $H_{2}$ is a trivial product state

$$
\left|\left\{g_{i j}=1\right\}\right\rangle,
$$

which is symmetric under the algebraic $(n-1)$-symmetry $G^{(n-1)}$. In the other limit $|J| \ll B \ll U$, the ground state of $H_{2}$ is a topologically ordered state (described by the $G$-gauge theory), breaking the algebraic $(n-1)$-symmetry $G^{(n-1)}$ spontaneously.

What is the global $G$ symmetry operator in the first model Eq. (79) mapped to? It is mapped to a global zero symmetry operator:

$$
\mathcal{U}_{h} H_{2}=H_{2} \mathcal{U}_{h}, \quad \mathcal{U}_{h}=\prod_{i} Q_{h}(i) .
$$

In particular, when the model has periodic boundary condition, this zero symmetry acts as $\mathcal{U}_{h}\left|g_{i j}\right\rangle=\left|h g_{i j} h^{-1}\right\rangle$. Thus the global symmetry is an inner automorphism of $G$, denoted as $\operatorname{Inn}(G)$. When the centralizer of $G$ is trivial, $\operatorname{Inn}(G) \cong G$.

Only when $G$ is Abelian, the global symmetry action in (87) reduces to claiming the boundary conditions or the twisted sectors of the model. For example, when $G=Z_{2}$ and $d=1$, it reduces to $U_{\widetilde{Z}_{2}}$ in $(11)$.

Furthermore, the symmetry generators of the algebraic $(n-1)$-symmetry $G^{(n-1)}$ and the zero-symmetry $\operatorname{Inn}(G)$ commute:

$$
W_{q}\left(S^{1}\right) \mathcal{U}_{h}=\mathcal{U}_{h} W_{q}\left(S^{1}\right) .
$$

In the limit $U \rightarrow+\infty$, the low-energy part of $H_{2}$ can be mapped to $H_{1}$ via the following duality and inverse duality map:

$$
g_{i j}=g_{i} g_{j}^{-1}, \quad\left(g_{i_{0}}\right)^{-1} g_{i}=\left(g_{i_{0} j} g_{j k} \cdots g_{l i}\right)^{-1},
$$

where $i_{0}$ is a fixed base point. Note that to map a configuration $g_{i}$ to a configuration $g_{i j}$ we need to pick a base point $i_{0}$ and a value $g_{i_{0}}$. Therefore, the above map is a $|G|$-to-one map. It maps the following $|G|$ configurations of $H_{1}$ (label by $h \in G$ ), $\left|\left\{h g_{i}\right\}\right\rangle$, into the same configuration of $H_{2},\left|\left\{g_{i j}\right\}\right\rangle$. Thus the spectrum of $H_{1}$ formed by $G$ invariant states, $|\Psi\rangle=U_{h}|\Psi\rangle$, is identical to the low-energy spectrum of $H_{2}$ below $U . H_{1}$ and $H_{2}$ have the same $G$-symmetric low-energy dynamics. In particular they have the same phase transition and critical point.

The $G$ symmetry-breaking phase of $H_{1}$ corresponds to the trivial phase of $H_{2}$ [which is the symmetric phase of the algebraic ( $n-1)$-symmetry $G^{(n-1)}$ ] and the $G$-symmetric phase of $H_{1}$ corresponds to the topologically ordered phase of $\mathrm{H}_{2}$ [which is the symmetry-breaking phase of the algebraic $(n-1)$-symmetry $\left.G^{(n-1)}\right][8,15]$. Now we see that the critical point at the symmetry-breaking transition neighbors a phase with $G$ zero symmetry and a phase with algebraic $(n-1)$ symmetry. Heuristically, the emergent symmetry at the critical point is the same or larger than the neighboring gapped phases. Thus the critical point has both the $G$ zero symmetry and the algebraic $(n-1)$-symmetry $G^{(n-1)}$. In other words, the critical point has a categorical symmetry $G \vee G^{(n-1)}$ which is the combination of the $G$ zero symmetry and the algebraic $(n-1)$-symmetry $G^{(n-1)}$. 


\section{B. Patch symmetry operators}

Now let us discuss the charges of the categorical symmetry in the model given by the previous two descriptions. Just as the case $G=Z_{2}$ discussed before, we can only create neutral charges, by patch operators. The 0 -symmetry patch operator creates conserved charges of $(n-1)$-symmetry; part of the conserved charges can be measured by the $(n-1)$-symmetry patch operator, and vice versa.

We start with the simple case that $n=1$. For a generic group, one set of patch operators, from site $i_{1}$ to site $i_{2}$, acting on a state $\left|\left\{g_{i j}\right\}\right\rangle$, is

$$
W_{q, \alpha \beta}\left(i_{1}, i_{2}\right)=\left(\prod_{i=i_{1}}^{i_{2}} R_{q}\left(g_{i j}\right)\right)_{\alpha \beta},
$$

where $\alpha, \beta$ runs from 1 to $n_{R}$, the dimension of the irreducible representation $R_{q}$ of $G$. The other set of patch operators is

$$
\mathcal{U}_{h}\left(i_{1}, i_{2}\right)=\prod_{i=i_{1}}^{i_{2}} Q_{h}(i) .
$$

These operators satisfy the following commutation relations with the ordering $i_{1}<i_{2}<i_{3}<i_{4}$, and a simplified notation $W_{q}\left(i_{1}, i_{3}\right)=W_{q, 13}, \mathcal{U}_{h}\left(i_{2}, i_{4}\right)=\mathcal{U}_{h, 24}$ [with the subscripts $\alpha, \beta$ of $W_{q}\left(l_{1}, l_{2}\right)$ suppressed]:

$$
\begin{aligned}
\operatorname{Tr}\left[W_{q, 13} \mathcal{U}_{h, 24}\left(W_{q, 13}\right)^{\dagger}\right] & =\chi^{q}\left(h^{-1}\right) \mathcal{U}_{h, 24}, \\
\operatorname{Tr}\left[\left(W_{q, 13}\right)^{\dagger} \mathcal{U}_{q, 24} W_{q, 13}\right] & =\chi^{q}(h) \mathcal{U}_{h, 24}, \\
\operatorname{Tr}\left[\left(W_{q, 24}\right) \mathcal{U}_{h, 13}\left(W_{q, 24}\right)^{\dagger}\right] & =\chi^{q}(h) \mathcal{U}_{h, 13}, \\
\operatorname{Tr}\left[\left(W_{q, 24}\right)^{\dagger} \mathcal{U}_{h, 13} W_{q, 24}\right] & =\chi^{q}\left(h^{-1}\right) \mathcal{U}_{h, 13} .
\end{aligned}
$$

where $\chi^{q}(h)$ is the character of $h$ in the $q$ representation. The character represents that the 0 -symmetry charge and the algebraic $(n-1)$-symmetry charge are mutually nonlocal.

More generally, for any $n$, the patch operator that creates the neutral charge for dual $(n-1)$-symmetry $G^{(n-1)}$ is defined on a $n$-dimensional patch (disk), $D^{n}$, and is given by the product of star terms: ${ }^{5}$

$$
\mathcal{U}_{h}\left(D^{n}\right)=\prod_{i \in D^{n}} Q_{h}(i)
$$

The $G^{(n-1)}$ neutral charge lives on the boundary of $D^{n}$, denoted as $\left(S^{n-1}\right)^{\vee}$, living on the dual lattice. Let us call it $s$. In particular, when $G$ is Abelian, $\mathcal{U}_{h}\left(D^{n}\right)$ acts trivially inside $D^{n}$. That is, $\mathcal{U}_{h}$ is in fact defined on the boundary of $D^{n}: 6$

$$
\begin{gathered}
\mathcal{U}_{h}\left[\left(S^{n-1}\right)^{\vee}\right]=\prod_{i j \in\left(S^{n-1}\right)^{\vee}} T_{h}(i j), \\
T_{h}(i j)\left|g_{i j}\right\rangle= \begin{cases}\left|h g_{i j}\right\rangle & i \in D^{n} \\
\left|g_{i j} h^{-1}\right\rangle & j \in D^{n}\end{cases}
\end{gathered}
$$

\footnotetext{
${ }^{5}$ In the low-energy sub-Hilbert space symmetric under the $\operatorname{Inn}(G)$ symmetry, $\mathcal{U}_{g}=1$. It follows that the patch operator is defined up to a conjugacy class of a representative $h \in G, \mathcal{U}_{g^{-1}} \mathcal{U}_{h}\left(D^{n}\right) \mathcal{U}_{g}=$ $\mathcal{U}_{\text {ghg-1 }}\left(D^{n}\right)$.

${ }^{6}$ Note that in the case $G$ is Abelian we can take $U_{h}\left[\left(S^{n-1}\right)^{\vee}\right]$ as the generator of a 1-symmetry. In the case that $G$ is Abelian $\sum_{h \in C_{a}} \mathcal{U}_{h}$, where the sum is over a conjugacy class of $a \in G$, has codimension 2 , relative to the spacetime dimension. They are the Gukov-Witten operators [59].
}

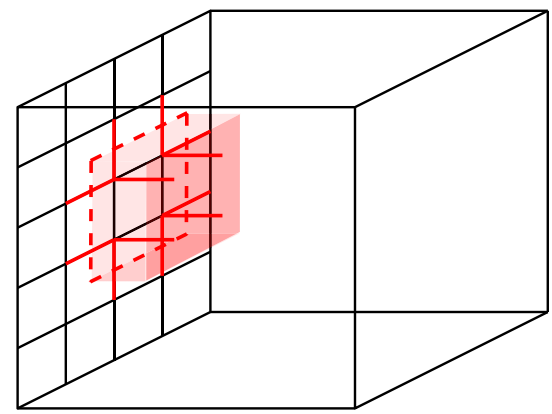

FIG. 3. A $Z_{2}^{(1)}$ neutral charge, an $s$ string on the $2 \mathrm{~d}$ boundary (red dashed loop), is created by a $Z_{2}$ membrane operator Eq. (57) in the $3 \mathrm{~d}$ bulk (red surface). It is only neutral when on the boundary. If we translate this membrane to the bulk, there are $U_{Z_{2}}\left(S^{1}\right)$ operators in the bulk that anticommute the membrane operator, justifying it as the $Z_{2}$ vortex topological excitation.

For $G=Z_{2}$ and $d=2$, we recover the $s$ string operator Eq. (53).

The other patch operator that creates conserved charges for the zero symmetry $G$ is $W_{q}\left(C_{i j}\right)$ defined on any open string $C_{i j}$. The zero symmetry charges are at the end-point $i$ and $j$ sites of the open string. Let us call them $e$ particles.

These charges can be thought of as the topological excitations in $(n+1) \mathrm{D}$ topological order [5,6,30,60,61]. The $e$ particle corresponds to the pointlike topological excitations, and the $s$ corresponds to the other topological excitations on the closed $\left(S^{n-1}\right)^{\vee}$ surface. For example, when $d=2$ and $G=Z_{2}, \mathcal{U}_{h}\left[\left(S^{1}\right)^{\vee}\right]$ is the closed $Z_{2}$ vortex string operator. This conserved charge of $\widetilde{Z}_{2}^{(1)}$ can be thought of as coming from the topological string excitation in $3+1$-dimensional $Z_{2}$ topological field theory, as discussed in Sec. III and illustrated in Fig. 3.

\section{Example of algebraic 1 symmetry $S_{3}^{(1)}$ in $2+1 D$ theories}

The simplest example where the algebraic symmetry is beyond a higher symmetry is in the $(n+1) \mathrm{D}$ model $(82)$ with $d=2$ and $G=S_{3}$. Here, $S_{3}=\left\langle s, r \mid s^{3}=r^{2}=1, r s r=s^{2}\right\rangle$ is the permutation group on three elements. The topologically ordered phase of (82) is described by $S_{3}$ gauge theory. There are eight types of anyonic excitations in the model. Their fusion rules are shown in Table I.

The model (82) has an algebraic 1-symmetry, which is denoted as $S_{3}^{(1)}$. The generators are two Wilson line operators [see Eq. (84)], labeled by the two nontrivial irreducible representations $a^{1}$ and $a^{2}$ of $S_{3}$. The ends of Wilson line operators create anions $a^{1}$ and $a^{2}$ the fusion of which is described in Table I. The product of Wilson line operators is given by the fusion of irreducible representations (i.e., the fusion of the anions $a^{1}$ and $a^{2}$ ):

$$
\begin{aligned}
& W^{a^{1}}\left(S^{1}\right) W^{a^{1}}\left(S^{1}\right)=\mathbb{1}, \\
& W^{a^{2}}\left(S^{1}\right) W^{a^{2}}\left(S^{1}\right)=\mathbb{1}+W^{a^{1}}\left(S^{1}\right)+W^{a^{2}}\left(S^{1}\right), \\
& W^{a^{1}}\left(S^{1}\right) W^{a^{2}}\left(S^{1}\right)=W^{a^{2}}\left(S^{1}\right) .
\end{aligned}
$$

The product of two $W^{a^{2}}\left(S^{1}\right)$ 's reveals that the symmetry is an algebraic $(n-1)$-symmetry beyond higher group. In general, 


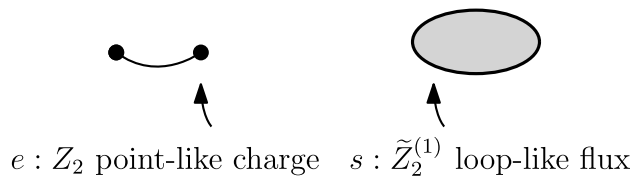

FIG. 4. The conservation (the fusion rule) of the $Z_{2}$ pointlike charge and $\widetilde{Z}_{2}^{(1)}$ looplike flux in the $3+1 \mathrm{D} Z_{2}$ gauge theory give rise to the categorical symmetry of the $2+1 \mathrm{D}$ lattice model $H_{1}(76)$. The mod-2 conservation of $Z_{2}$ charge $e$ gives rise to the $Z_{2}$ zero symmetry. The mod-2 conservation of $Z_{2}^{(1)}$ flux $s$ gives rise to the $Z_{2}^{(1)}$ one symmetry. $e$ and $s$ have a mutual $\pi$ statistics between them.

if $G$ is non-Abelian, $G^{(n-1)}$ is an algebraic $(n-1)$-symmetry beyond higher group.

\section{Holographic point of view}

Let us start with the lattice model $H_{1}$ (76) with a finite zero symmetry $G$. We would like to study the $G$ symmetry within the restricted symmetric sub-Hilbert space. In the symmetric sub-Hilbert space, the $G$ symmetry transformation (79) will be trivial. It appears that we cannot see the $G$ symmetry. But we can see the $G$ symmetry via pointlike excitations in a finite region, which carry nontrivial representations of $G$. The nontrivial fusion of $G$ representations (in particular, the fusion channel of nontrivial representations into a trivial representation) signifies the $G 0$-symmetry in the symmetric sub-Hilbert space. Thus, restricting to the symmetric subHilbert space forces us to view the $G$ symmetry via the fusion category of the symmetry charges. This is the categorical view of symmetry [62].

The lattice model $H_{1}$ (76), when restricted to the symmetric sub-Hilbert space, has a gravitational anomaly. This is because the symmetric sub-Hilbert space $\mathcal{V}_{\text {symm }}$ does not have a tensor product decomposition $\mathcal{V}_{\text {symm }} \neq \otimes_{i} \mathcal{V}_{i}$, in terms of the local Hilbert space $\mathcal{V}_{i}$ on each site. This suggests that the fusion category of the symmetry charges is anomalous [30,55], i.e., the fusion category can only be realized at a boundary of a topological order in one higher dimension. Indeed, the model $H_{1}$ (76), when restricted to the symmetric sub-Hilbert space, can be viewed as a boundary of $G$-gauge theory in one higher dimension, where a simple example is discussed in Sec. IID [31]. The gauge charges in the $G$-gauge theory also carry representations of $G$. The nontrivial fusion of $G$ representations gives rise to the $G 0$-symmetry both in the bulk and at the boundary. This is how the finite 0-symmetry $G$ in the model $H_{1}$ (76) appears via the $G$-gauge theory in one higher dimension.

But the $G$-gauge theory also has other excitations (such as the gauge flux-codimension-2 excitations), which also fuse in a nontrivial way and give rise to additional symmetry to the lattice model $H_{1}$ (76). So the complete symmetry of the lattice model $H_{1}$ (76) is given by the nontrivial fusion of all the excitations (see Fig. 4). Such a complete symmetry is called categorical symmetry of the lattice model $H_{1}$ (76) (when restricted to the symmetric sub-Hilbert space). The categorical symmetry is fully characterized by the $G$-gauge theory in one higher dimension. The data in one higher dimension include gauge charges, gauge fluxes, their fusion rules, and the mutually nonlocal property. The set of data is realized on the boundary as the global symmetry charges, the global algebraic higher symmetry charges, their fusion, as well as their mutual statistics. This is the holographic understanding of the categorical symmetry. Compared to our patch-operator understanding of the categorical symmetry discussed in Secs. II B and V B, the holographic view reveals the essence of the categorical symmetry more clearly.

Let us rephase the above holographic point of view using a categorical language (for details see Ref. [8] and Appendix A). The braiding and fusion of the particles carrying $G$ representations are described by the fusion $n$-category $n \mathcal{R} \operatorname{ep}(G)$. Every fusion higher category can be mapped into a braided fusion higher category by a $Z_{1}$ functor, called the $Z_{1}$ center in this paper [see Eq. (102), and, for a physical description, see, for example, Ref. [8]]. The $Z_{1}$ center of the fusion $n$-category $n \mathcal{R} \operatorname{ep}(G)$ is denoted as $Z_{1}[n \mathcal{R} \operatorname{ep}(G)]$, which is a braided fusion $n$-category. In fact, $Z_{1}[n \mathcal{R} \operatorname{ep}(G)]$ describes the excitations in the $G$-gauge theory in one higher dimension [i.e., in $(d+2)$-dimensional spacetime] and is denoted as $\mathcal{G}_{G}^{d}$. In other words, $\mathcal{G}_{G}^{d}=Z_{1}[n \mathcal{R} \operatorname{ep}(G)][8,63]$.

Therefore, for a system with symmetry described by a fusion $n$-category $n \mathcal{R} \operatorname{ep}(G)$ (which is nothing but the $G 0$ symmetry), to find its categorical symmetry is to find the $Z_{1}$ center of $n \mathcal{R} \operatorname{ep}(G): Z_{1}[n \mathcal{R} \operatorname{ep}(G)] . Z_{1}[n \mathcal{R} \operatorname{ep}(G)]$ describes the excitations in the $G$-gauge theory in one higher dimension. This is the holographic point of view of the categorical symmetry.

We stress that the lattice model $H_{1}$ (76) (when restricted to the symmetric sub-Hilbert space) has the full categorical symmetry, but its ground states may spontaneously break part of the categorical symmetry. Those different ground states correspond to different boundaries of the $G$-gauge theory. Since a gapped boundary of $G$-gauge theory always comes from condensation of gauge charges, or gauge flux, or some combination of them, a gapped boundary always spontaneously breaks some part of the categorical symmetry. Therefore, the gapped ground states of $H_{1}$ always spontaneously break some part of the categorical symmetry. Because the gauge charge and gauge flux have nontrivial mutual statistics between them, we cannot condense all gauge charges and gauge fluxes simultaneously. Therefore, any ground states of the lattice model $H_{1}$ (76) cannot break the categorical symmetry completely.

The $G$-gauge theory has a gapless boundary if none of the gauge charges or gauge fluxes is condensed. Such a boundary does not break the categorical symmetry. Thus the lattice model $H_{1}$ (76) has a gapless ground state where the categorical symmetry is not spontaneously broken. This gapless state should correspond to the critical point of the Landau $G$ symmetry-breaking transition. The above discussions also apply to the dual model $H_{2}(82)$.

\section{EMERGENCE OF ALGEBRAIC HIGHER SYMMETRY}

We have seen that a $G 0$-symmetry in $n$-dimensional space can be fully characterized by a fusion $n$-category $n \mathcal{R} \operatorname{ep}(G)$, describing the fusion of the charge objects of the 
$G$ symmetry. In fact, the charge objects described by $n \mathcal{R} \operatorname{ep}(G)$ are nothing but the excitations on top of a product state with the $G$ symmetry. To be precise, $n \mathcal{R} \operatorname{ep}(G)$ describes the types of the excitations, which are the equivalence classes under the $G$ symmetry preserving deformations (i.e., two excitations are equivalent if they can deform into each other smoothly without breaking the symmetry). This is why $n \mathcal{R} \operatorname{ep}(G)$ depends on the symmetry $G$, despite it describes excitations in a trivial product state.

Similarly, the algebraic $(n-1)$-symmetry $G^{(n-1)}$ in the lattice model Eq. (82) is fully characterized by a fusion $n$ category $n \mathcal{V e c}{ }_{G}$, describing the fusion of the charge objects of the $G^{(n-1)}$-symmetry. Again, $n \mathcal{V} \operatorname{ec}_{G}$ describes the types of the excitations on top of a product state with the $G^{(n-1)}$ symmetry. Now types are the equivalence classes under the $G^{(n-1)}$-symmetry preserving deformations.

This result can be generalized. Consider a lattice model with an algebraic higher symmetry in $n$-dimensional space, which has a symmetric product state as its ground state [such as Eq. (86)]. The type of the excitations on top of the product state is described by a fusion $n$-category $\mathcal{R}$, and the algebraic higher symmetry is fully characterized by $\mathcal{R}$. So we will refer to an algebraic higher symmetry as $\mathcal{R}$.

We would like to remark that the fusion higher category $\mathcal{R}$ describing excitations on top of a product state is a special class of fusion higher category, called the local fusion higher category. Actually, describing types of excitations on top of a symmetric product state is the defining property of a local fusion higher category. We believe that local fusion n-categories classify algebraic higher symmetries in n-dimensional space [8].

Now, let us consider a lattice theory or a field theory in $n$-dimensional space, the low-energy excitations of which happen to be described by a local fusion $n$-category $\mathcal{R}$. If we ignore all the high-energy excitations and pretend $\mathcal{R}$ are only excitations, then we can pretend $\mathcal{R}$ to be the excitations in a product state with the algebraic higher symmetry $\mathcal{R}$. In this way, we say that the theory has an emergent algebraic higher symmetry $\mathcal{R}$, and we can regard the system to be in a trivial $\mathcal{R}$-symmetric phase.

Let us elaborate with some examples of emergent algebraic higher symmetries. The first is the model with a finite zero symmetry $G$ in $n$-dimensional space, which we now discuss using the point of view of the local fusion higher category. If the ground state of the model is a product state with $G$ symmetry, then the excitations will be pointlike and are labeled by the irreducible representations of $G$. Those excitations are described by a local fusion $n$-category $n \mathcal{R} \operatorname{ep}(G)$. Thus the $G$ zero symmetry can also be denoted as $n \mathcal{R} \operatorname{ep}(G)$ symmetry. If the model is in the spontaneous symmetry-breaking phase, the ground states will be degenerate and are labeled by the ground elements $g \in G$. The excitations will be $(n-1)$-dimensional domain walls between different degenerate ground states. Those domain-wall excitations are labeled by pairs $\left(g_{1}, g_{2}\right)$ if the domain wall connects the ground state $g_{1}$ and the ground state $g_{2}$. Under a symmetry transformation $g \in G$, the domain wall transforms as $\left(g_{1}, g_{2}\right) \rightarrow\left(g g_{1}, g g_{1}\right)$. We say $\left(g_{1}, g_{2}\right)$ and $\left(g g_{1}, g g_{1}\right)$ are equivalent. The equivalent classes of domain walls (i.e., symmetrized domain walls) are labeled by a single group element $h=g_{1}^{-1} g_{2}$. Those excitations are described by a fusion $n$-category $n \mathcal{V e c}{ }_{G}$. It turns out that $n \mathcal{V e c}{ }_{G}$ is also a local fusion $n$-category [8]. The algebraic higher symmetry $n \mathcal{V e c}_{G}$ is nothing but the algebraic $(n-1)$-symmetry $G^{(n-1)}$ generated by Wilson loop operators that we discussed before.

The second example of the $2 \mathrm{~d}$ lattice model has $S_{3}=Z_{3} \rtimes$ $Z_{2}$ zero symmetry. We have a phase with $S_{3}$ zero symmetry. We have another phase that spontaneously breaks the $S_{3}$ zero symmetry. This phase has an emergent $S_{3}^{(1)}$ algebraic 1 -symmetry. We also have some other phases that break different symmetries and thus have different emergent algebraic higher symmetries. All those phases and their emergent algebraic higher symmetries are listed as follows: (1) the $S_{3}$-symmetric phase, the pointlike charges of which are

$$
R_{S_{3}}=2 \mathcal{R e p}\left(S_{3}\right)=\left\{11, p_{1}, p_{2}\right\} ;
$$

(2) the $Z_{2}$ (charge conjugation) spontaneous symmetrybreaking phase with $Z_{3}$ symmetry, the pointlike and stringlike excitations of which are

$$
R_{Z_{3}, Z_{2}^{(1)}}=\{11, p, \bar{p}, s\},
$$

which includes $Z_{3}$ charges $p, \bar{p}$ (pointlike) and $Z_{2}$ domain wall $s$ (stringlike); (3) the $Z_{3}$ spontaneous symmetry-breaking phase with $Z_{2}$ symmetry,

$$
R_{Z_{2}, Z_{3}^{(1)}}=\{11, p, s, \bar{s}\},
$$

which includes $Z_{2}$ charge $p$ (pointlike) and $Z_{3}$ domain wall $s, \bar{s}$ (stringlike); and (4) the $S_{3}$ spontaneous symmetry-breaking phase, the stringlike excitations of which are labeled group elements,

$$
R_{S_{3}^{(1)}}=2 \mathcal{V e c}_{S_{3}}=\left\{s_{h} \mid h \in G\right\} .
$$

$R_{S_{3}}=2 \mathcal{R e p}\left(S_{3}\right)$ and $R_{S_{3}^{(1)}}=2 \mathcal{V e c}_{S_{3}}$ are local fusion 2categories, since they describe excitations on top of symmetric product states, as explicitly shown in Sec. V. They correspond to algebraic higher symmetries: the 0 -symmetry $S_{3}$ and the algebraic 1-symmetry $S_{3}^{(1)}$. Using the results in Ref. [8], we find that $R_{Z_{2}, Z_{3}^{(1)}}$ and $R_{Z_{3}, Z_{2}^{(1)}}$ are also local fusion 2-categories, and they also correspond to two algebraic higher symmetries. The algebraic higher symmetry $R_{Z_{2}, Z_{3}^{(1)}}$ contains a 0 symmetry $Z_{2}$ (the conservation of the $Z_{2}$ charges) and a 1-symmetry $Z_{3}^{(1)}$ (the conservation of the $Z_{3}$ domain walls). The algebraic higher symmetry $R_{Z_{3}, Z_{2}^{(1)}}$ contains a 0 -symmetry $Z_{3}$ (the conservation of the $Z_{3}$ charges) and a 1-symmetry $Z_{2}^{(1)}$ (the conservation of the $Z_{2}$ domain walls).

Those four algebraic higher symmetries form two dual pairs: $\left(2 \mathcal{R} \operatorname{ep}\left(S_{3}\right), 2 \mathcal{V} \mathrm{ec}_{S_{3}}\right)$ and $\left(R_{Z_{2}, Z_{3}^{(1)}}, R_{Z_{3}, Z_{2}^{(1)}}\right)$. Moreover, the $Z_{1}$ center of all the above $\mathcal{R}$ 's is the same $Z_{1}(R)=\mathcal{G}_{S_{3}}^{2}$, the same category that describes the topological data of $3 \mathrm{~d} S_{3}$ topological order, which characterizes the $S_{3} \vee S_{3}^{(1)}$ categorical symmetry.

In fact, all the four phases discussed above have the same emergent categorical symmetry $S_{3} \vee S_{3}^{(1)}$. But in different phases the categorical symmetry is spontaneously broken in different ways. It turns out that, to understand the emergent algebraic higher symmetry, it is better to understand the emergent categorical symmetry first. Then, the emergent algebraic higher symmetry is just the unbroken part of the emergent categorical symmetry. In the next section, we use this point 
of view to understand the emergent categorical symmetry and emergent algebraic higher symmetry in a more general setting.

\section{EMERGENCE OF CATEGORICAL SYMMETRY (AND ALGEBRAIC HIGHER SYMMETRY)}

In this section, we consider a lattice theory or a field theory in $n$-dimensional space, the low-energy excitations of which are described by a fusion $n$-category $\mathcal{C}$. Some excitations in $\mathcal{C}$ may correspond to charge objects of a certain symmetry, and other excitations correspond to topological excitations not associated with symmetry. Here we ignore all the high-energy excitations and pretend $\mathcal{C}$ are the only excitations. Moreover, we use the categorical point of view of symmetry, i.e., we view all the charge objects as topological excitations, and ignore their symmetry origin. This is possible since the symmetry is fully encoded in the fusion of the charge objects. Now, we would like to ask the following: what is the emergent algebraic higher symmetry in a theory with low-energy excitations $\mathcal{C}$ ? First we would like to understand what is the emergent categorical symmetry in a theory with low-energy excitations $\mathcal{C}$.

Let us consider an example of a $2+1 \mathrm{D}$ product state with a $Z_{2}$ symmetry. The state has pointlike excitations $\mathcal{C}_{Z_{2} \text {-sym }}=$ $\{11, e\}$, where 11 has $Z_{2}$ charge zero, and $e$ has $Z_{2}$ charge one. Since the Hamiltonian has $Z_{2}$ symmetry, $\mathcal{C}_{Z_{2} \text {-sym }}=\{11, e\}$ should give rise to the $Z_{2}$ symmetry. The second example is the $Z_{2}$ topological order (described by $Z_{2}$ gauge theory) without any symmetry. The topological phase has pointlike excitations $\mathcal{C}_{Z_{2} \text {-top }}=\{11, e, m, f\}$. Since the Hamiltonian has no symmetry, $\mathcal{C}_{Z_{2} \text {-top }}=\{11, e, m, f\}$ should not give rise to any symmetry.

When viewed as two fusion 2-categories describing topological excitations in $2 \mathrm{~d}$ topological orders, why does $\mathcal{C}_{Z_{2} \text {-sym }}$ give rise to symmetry while $\mathcal{C}_{Z_{2} \text {-top }}$ gives rise to no symmetry? To see their difference, here we would like to introduce the notion of a gravitational anomaly. Conventionally, the gravitational anomaly refers to a noninvariance of the pathintegral measure under the diffeomorphism transformations of the spacetime manifold. Here, following Refs. [30,55], we define gravitational anomaly differently, as the obstruction to regularize the theory by a local lattice bosonic model without symmetry in the same dimension. We ask whether there exists a local lattice bosonic model without symmetry in the same dimension, the complete excitations of which reproduce the fusion category $\mathcal{C}$. If such a lattice model exists, then the fusion category $\mathcal{C}$ is free of the gravitational anomaly. If the lattice regularization without symmetry does not exist, then the fusion category $\mathcal{C}$ has a gravitational anomaly. It turns out that $\mathcal{C}_{Z_{2} \text {-top }}$ has no gravitational anomaly, while $\mathcal{C}_{Z_{2} \text {-sym }}$ has a gravitational anomaly. One may say that $\mathcal{C}_{Z_{2} \text {-sym }}$ can be realized as excitations in a lattice model, and should be anomaly-free. However, the lattice regularization of $\mathcal{C}_{Z_{2} \text {-sym }}$ requires a $Z_{2}$ symmetry. $\mathcal{C}_{Z_{2} \text {-sym }}$ has no lattice regularization without symmetry, and thus has a gravitational anomaly.

The above examples reveals a general property: if the excitations are described by anomaly-free fusion higher category $\mathcal{C}$, then there is no emergent symmetry. Here, the emergent symmetries are global symmetries that can be beyond grouplike. ${ }^{7}$ If $\mathcal{C}$ is anomalous, then there is an emergent symmetry. ${ }^{8}$ We see that emergent symmetry essentially implies a gravitational anomaly. So to understand emergent symmetry we need to understand gravitational anomaly.

But in the above we just defined what is the "no gravitational anomaly" as the existence of lattice regularization in the same dimension. We did not define what is the gravitational anomaly. To define what is the gravitational anomaly, we rely on the following conjecture, the holographic principle of topological order $[30,64,65]$ : The excitations in $n$-dimensional space described by a fusion $n$-category $\mathcal{C}$ can always be realized at a boundary of an anomaly-free topological order (denoted as $M$ ) in one higher dimension. Moreover, the topological order $M$ is uniquely determined by $\mathcal{C}$, and we denote this map from $\mathcal{C}$ and $M$ as $M=\operatorname{bulk}(\mathcal{C})$. Using the holographic principle, we can rephrase the anomaly-free condition for a fusion higher category $\mathcal{C}$ as

$$
\operatorname{bulk}(\mathcal{C})=11,
$$

where 11 denotes the trivial topological order (i.e., a product state with no symmetry). This is because if $\mathcal{C}$ can be realized by a boundary of a product state in a lattice model in one higher dimension we can always remove the bulk product state and conclude that $\mathcal{C}$ can be realized by a lattice model in the same dimension. Thus the holographic principle can tell us when there exists a gravitational anomaly. Furthermore, the holographic principle gives a way to regularize the anomalous theory $\mathcal{C}$ on a $n+1$-dimensional lattice, and $\mathcal{C}$ is realized as one low-energy phase of a boundary of the lattice model. Thus the holographic principle tells us what is the gravitational anomaly: a gravitational anomaly is a topological order in one higher dimension [30,64,65].

Should we view the bulk topological order $M$ (the gravitational anomaly) as the emergent symmetry? Maybe not. The emergent symmetry should be related to conservation laws encoded by fusion rules, or more precisely a fusion higher category. In fact, from the bulk topological order $M$ in $(n+$ 1)-dimensional space, we can get a fusion $(n+1)$-category $\mathbb{M}$ describing its excitations. Since the bulk topological order $M$ is anomaly free all the codimension-1 excitations are descendent (i.e., formed by codimension-2 and higher excitations). We can drop the codimension- 1 excitations, which turns the fusion $(n+1)$-category $\mathbb{M}$ into a braided fusion $n$-category $\mathcal{M}$. This turns the map from $\mathcal{C}$ to $M$, where $M=\operatorname{bulk}(\mathcal{C})$, into a map from $\mathcal{C}$ to $\mathcal{M}$ :

$$
\mathcal{M}=Z_{1}(\mathcal{C}) .
$$

So we should view the braided fusion $n$-category $\mathcal{M}$ as the emergent symmetry $\mathcal{C}$. Since $\mathcal{M}$ describes the excitations in a topological order in one higher dimension where $\mathcal{C}$ is

\footnotetext{
${ }^{7}$ By "beyond grouplike," we allow at least the following two kinds of generalizations: first the multiplication of symmetry generators is given by an algebra that is not grouplike; second the charges of the symmetry can be mutually nonlocal.

${ }^{8}$ When the emergent symmetry is a global symmetry of a (finite) group $G$, the theory is a low-energy theory of either a symmetry protected phase or a spontaneously symmetry-breaking phase on a local lattice bosonic model with a global symmetry $G$.
} 
realized as a boundary, we see that $\mathcal{M}$ is actually the emergent categorical symmetry.

Now, we can say that the fusion 2 category $\mathcal{C}_{Z_{2} \text {-top }}=$ $\{11, e, m, f\}$ that describes the excitations in the $2+1 \mathrm{D} Z_{2}$ topological order has no emergent categorical symmetry since $Z_{1}\left(\mathcal{C}_{Z_{2} \text {-top }}\right)=\{11\}$. Similarly, the fusion 2 category $\mathcal{C}_{0}=\{11\}$ that describes the excitations in the $2+1 \mathrm{D}$ product state with no symmetry has no emergent categorical symmetry since $Z_{1}\left(\mathcal{C}_{0}\right)=\{11\}$, since both phases have no categorical symmetry. We can find a lattice model with no symmetry in which the above two phases can transform into each other via phase transitions. As there is no global symmetry to be concerned, we just turn off the Hamiltonian for $Z_{2}$ topological order and turn on another for the product state.

On the other hand, the fusion 2 category $\mathcal{C}_{Z_{2} \text {-sym }}=\{11, e\}$ that describes the excitations in the $2+1 \mathrm{D} Z_{2}$-symmetric product state has an emergent categorical symmetry described by $Z_{1}\left(\mathcal{C}_{Z_{2} \text {-sym }}\right)$. In fact, $Z_{1}\left(\mathcal{C}_{Z_{2} \text {-sym }}\right)$ is the braided fusion 2 category (denoted as $\mathcal{G}_{Z_{2}}^{2}$ ) describing the excitations in a $3+1 \mathrm{D}$ $Z_{2}$ gauge theory. Therefore, $\mathcal{C}_{Z_{2} \text {-sym }}=\{11, e\}$ has an emergent categorical symmetry $\mathcal{G}_{Z_{2}}^{2}=Z_{1}\left(\mathcal{C}_{Z_{2} \text {-sym }}\right)$. In this case, we cannot connect a phase described by the fusion 2 category $\mathcal{C}_{\mathrm{Z}_{2} \text {-sym }}=\{11, e\}$ to a phase described by the fusion 2 category $\mathcal{C}_{0}=\{11\}$.

The above result sounds counterintuitive, since $\mathcal{C}_{Z_{2} \text {-sym }}=$ $\{11, e\}$ can be realized by a $Z_{2}$-symmetric product state and $\mathcal{C}_{0}=\{11\}$ can be realized by a product state with no symmetry. Two product states should be in the same phase and are connected by phase transitions (actually connected by zero phase transition). When we say $Z_{2}$-symmetric product state, we also specify the deformation class of the Hamiltonians, which are all required to have the $Z_{2}$ symmetry. In the phase diagram of the $Z_{2}$-symmetric Hamiltonians, there is no phase the excitations of which are given by $\mathcal{C}_{0}=\{11\}$, but there is a phase the excitations of whcih are given by $\mathcal{C}_{Z_{2} \text {-sym }}=\{11, e\}$. This is what we mean by "a phase described by the fusion 2 category $\mathcal{C}_{Z_{2} \text {-sym }}=\{11, e\}$ is not connected to a phase described by the fusion 2-category $\mathcal{C}_{0}=\{11\}$ ”. In general, if low-energy excitation $\mathcal{C}$ has nontrivial categorical symmetry $Z_{1}(\mathcal{C})$, then any gapped phase formed by condensing those low-energy excitations cannot be a trivial phase with excitation $\{\mathbb{1 1}\}$. This comes from the knowledge that $Z_{1}(\mathcal{C}) \neq Z_{1}(\{11\})$.

We see that the emergent categorical symmetry can constrain the possible phases and phase transitions, just like the usual symmetry does. This represents one of the most important applications of categorical symmetry (see Sec. VII A).

Let us discuss more examples of emergent categorical symmetry using the $2+1 \mathrm{D} Z_{2}$ topological order $[66,67]$. The $Z_{2}$ topological order has a trivial excitation $\mathbb{1 l}$ and three nontrivial excitations $e, m$, and $f$ with $\bmod 2$ conservation. $e$ and $m$ are bosons and $f$ is a fermion, and they fuse as $e \otimes m=f$. If the low-energy excitations are $R=\{11, e\}$ (and $m$ and $f$ are assume to have very high energies), then $\mathcal{R}$ is a local fusion 2-category $R=2 \mathcal{R} \operatorname{ep}\left(Z_{2}\right)$, which describes an anomaly-free $Z_{2} 0$-symmetry. The system also has an emergent categorical symmetry described by $Z_{1}(R)=Z_{1}\left[2 \mathcal{R} \operatorname{ep}\left(Z_{2}\right)\right]=\mathcal{G}_{Z_{2}}^{2}$, which is the braided fusion category describing the excitations in $3+$ $1 \mathrm{D} Z_{2}$-gauge theory. Such a categorical symmetry contains a $Z_{2} 0$-symmetry and a $Z_{2}^{(1)} 1$-symmetry and is denoted as $Z_{2} \vee$
$Z_{2}^{(1)}$. We may also say that the low-energy physics of $\{11, e\}$ is controlled by the emergent categorical symmetry $Z_{2} \vee Z_{2}^{(1)}$. For example, the $e$ excitations may condense and drive the system to another gapped phase that spontaneously breaks the $Z_{2}$ symmetry but has the $Z_{2}^{(1)} 1$ symmetry. The excitations in the new phase are described by $\{11, s\}=2 \mathcal{V e c}_{Z_{2}}$, where $s$ is a stringlike excitation with $Z_{2}$ fusion $s \otimes s=\mathbb{1}$. Such a gapped phase is possible since it has the same categorical symmetry $Z_{1}\left(2 \mathcal{V e c}_{Z_{2}}\right)=Z_{1}\left[2 \mathcal{R} \operatorname{ep}\left(Z_{2}\right)\right]$. At the transition between the phases, we have a gapless critical point formed by $\{11, e\}$ (or equivalently formed by $\{11, s\}$ ). which has the unbroken categorical symmetry $Z_{2} \vee Z_{2}^{(1)}$. It requires that both the $Z_{2}$ charges $e$ and $Z_{2}^{(1)}$ charges $s$ are not condensed at the critical point.

If the low-energy excitations are $\mathcal{C}=\{11, f\}$, [which is a fusion 2-category denoted as $\left.2 \mathcal{R} \operatorname{ep}\left(Z_{2}^{f}\right)\right]$, then $\mathcal{C}$ is not a local fusion higher category. The emergent categorical symmetry is described by $Z_{1}(\mathcal{C})=Z_{1}\left[2 \mathcal{R} \operatorname{ep}\left(Z_{2}^{f}\right)\right]$, which is the braided fusion category describing the excitations in a twisted $3+1 D$ $Z_{2}^{f}$-gauge theory where the $Z_{2}^{f}$ charge is a fermion [25]. Such a categorical symmetry contains a $Z_{2}^{f} 0$-symmetry (the fermion number parity) and a $\widetilde{Z}_{2}^{(1)} 1$-symmetry, and is denoted as $Z_{2}^{f} \vee Z_{2}^{(1)}$. The categorical symmetry controls the low-energy dynamics of $\{11, f\}$, which can be simulated by the boundary of the twisted $3+1 \mathrm{D} Z_{2}^{f}$-gauge theory. For example, we cannot have a phase that spontaneously breaks the fermionic $Z_{2}^{f}$ symmetry. Also, the categorical symmetry $Z_{2}^{f} \vee Z_{2}^{(1)}$ is an example that there is no corresponding algebraic higher symmetry $\mathcal{R}$, i.e., there is no local fusion 2 category $\mathcal{R}$, and satisfies $Z_{1}(R)=Z_{1}\left[2 \mathcal{R} \operatorname{ep}\left(Z_{2}^{f}\right)\right]$. Physically, this implies that there is no $2+1 \mathrm{D}$ bosonic system, with or without symmetry, the gapped state of which gives rise to the excitations described by $\mathcal{C}=\{11, f\}$.

In summary, for a system with low-energy excitations described by a fusion category $\mathcal{C}$, when $\mathcal{C}$ is a local one, the system has an emergent algebraic higher symmetry. In general, the largest emergent symmetry is the categorical symmetry given by $Z_{1}(\mathcal{C})$. Phases that have the same categorical symmetries are connected through phase transitions, since each of them is a phase spontaneously breaking part of the same categorical symmetry. Starting from the critical point that has the full category symmetry, the system can break different parts of the category symmetry and drive a transition to those spontaneous symmetry-breaking phases.

\section{A. Categorical symmetry, anomaly, and duality}

Let us consider two field theories ${ }^{9}$ the low-energy excitations of which are described by two fusion higher categories

\footnotetext{
${ }^{9}$ Here, by field theory, we mean that the UV regularization is not specified. In particular, when we say two phases in two field theories are connected by phase transitions, we mean that there exist UV regularizations (such as lattice models) for each field theory, and for such regularized field theories the two phases are connected by phase transitions. (There may be other different UV regularizations where the two phases are not connected by phase transitions.)
} 
$\mathcal{C}_{1}$ and $\mathcal{C}_{2}$, respectively. The two field theories may have different algebraic higher symmetries, and $\mathcal{C}_{1}$ and $\mathcal{C}_{2}$ may contain the charge objects of those algebraic higher symmetries. We would like to ask the following questions. Can the two theories be connected through phase transitions? Can we use one theory to simulate the other theory? Are the two theories dual to each other? In fact, the above three questions are the same question, since the following five statements are equivalent. ${ }^{10}$

(1) The two theories describe phases which are connected through phase transitions and other phases.

(2) The two theories can simulate each other.

(3) The two theories are dual to each other.

(4) The two theories have the same anomaly.

(5) The two theories have the same categorical symmetry $Z_{1}\left(\mathcal{C}_{1}\right)=Z_{1}\left(\mathcal{C}_{2}\right)$.

References [30,55] pointed out that the anomaly is simply topological/symmetry protected topological order in one higher dimension. In light of this view of anomaly, $Z_{1}\left(\mathcal{C}_{1}\right)$ and $Z_{1}\left(\mathcal{C}_{2}\right)$ simply correspond to the anomalies of the two theories, and $Z_{1}\left(\mathcal{C}_{1}\right)=Z_{1}\left(\mathcal{C}_{2}\right)$ is simply the anomaly matching condition. We would like to remark that $Z_{1}\left(\mathcal{C}_{1}\right)$ and $Z_{1}\left(\mathcal{C}_{2}\right)$ are in general noninvertible anomalies [31].

However, to be more precise, we should replace $Z_{1}\left(\mathcal{C}_{1}\right)$ and $Z_{1}\left(\mathcal{C}_{2}\right)$ in the above by the bulk topological orders of $\mathcal{C}_{1}$ and $\mathcal{C}_{2}$, which are denoted as $\operatorname{bulk}\left(\mathcal{C}_{1}\right)$ and $\operatorname{bulk}\left(\mathcal{C}_{2}\right)$, respectively. The anomaly matching condition is really bulk $\left(\mathcal{C}_{1}\right)=$ bulk $\left(\mathcal{C}_{2}\right)$. Such a condition implies that $\mathcal{C}_{1}$ and $\mathcal{C}_{2}$ can be viewed as two boundaries of the same bulk topological order. We know that two theories that are boundary theories of the same bulk topological order can simulate each other.

We remark that $Z_{1}\left(\mathcal{C}_{1}\right)$ describes the excitations in the bulk topological order bulk $\left(\mathcal{C}_{1}\right) . Z_{1}\left(\mathcal{C}_{1}\right)$ contains a little less information than the bulk topological order $\operatorname{bulk}\left(\mathcal{C}_{1}\right)$ : two topological orders differing by an invertible topological order have the same excitations.

We know that symmetry-breaking transitions are induced by the condensation of charge objects of the symmetry, while topological phase transitions are induced by condensing topological excitations. But in our setup the charge objects and topological excitations are treated on an equal footing, and thus symmetry-breaking transitions and topological transitions are treated on an equal footing. So the emergence of categorical symmetry happens at both symmetry-breaking transitions and topological transitions, as well as their mixtures.

As an application, we may consider a $2+1 \mathrm{D}$ field theory with a $Z_{2}$ zero symmetry and another $2+1 \mathrm{D}$ field theory with a $Z_{2}^{(1)} 1$-symmetry. Both symmetries give rise to the same categorical symmetry (i.e., the same bulk topological order), which is described by the $3+1 \mathrm{D} Z_{2}$ gauge theory. As a result, the two theories have the same gravitational anomaly. Then, the two theories can be connected by phase transitions, can simulate each other, and are dual to each other, even if we do not explicitly break the two symmetries (i.e., under the constraint of the two symmetries).

\footnotetext{
${ }^{10}$ See, for example, Ref. [76], for the recent development of simulating lattice gauge theories in dual variables with defects included.
}

\section{B. Example: Higgs and confinement transition in $3+1 \mathrm{D} Z_{2}$ gauge theory}

Let us discuss the simple example of $3+1 \mathrm{D} Z_{2}$ topological order to illustrate the above general results. In the first case, we choose the low-energy subcategory $R_{p}$ to be the one formed by all the pointlike excitations, i.e., the $Z_{2}$ charges [denoted as $\left.3 \mathcal{R} \operatorname{ep}\left(Z_{2}\right)\right]$. We assume all other excitations (such as gauge flux) to have infinite energy. In this case, we can focus only on excitations described by $R_{p}=3 \mathcal{R} \operatorname{ep}\left(Z_{2}\right)$, and our system can be viewed as a system with $Z_{2}$ zero symmetry. Our previous discussions on the $G$-symmetric system will apply. In particular, we have an (emergent) categorical symmetry characterized by $Z_{1}\left(R_{p}\right)=\mathcal{G}_{Z_{2}}^{3}$, which is a $Z_{2}$-gauge theory in $(4+1) \mathrm{D}$. The categorical symmetry contains $Z_{2}$ zero symmetry from the fusion of the pointlike $Z_{2}$ charges in the (4+1)-dimensional $Z_{2}$ gauge theory. The categorical symmetry also contains $Z_{2}^{(2)}$ two symmetry from the fusion of the membranelike $Z_{2}$ flux in the $4+1 \mathrm{D} Z_{2}$-gauge theory. The condensation of the $Z_{2}$ charge induces a Higgs transition from the $3+1 \mathrm{D} Z_{2}$ topological order to trivial order. The critical point of the Higgs transition has the $Z_{2} \vee Z_{2}^{(2)}$ categorical symmetry. In fact, such a critical point is the same as the $Z_{2}$ symmetry-breaking critical point discussed before.

In the second case, we choose the low-energy subcategory $R_{s}$ to be the one formed by the pure stringlike excitations, i.e., the $Z_{2}$ flux lines. We assume all other excitations (such as gauge charges) to have infinite energy. After ignoring other excitations, the only excitations are described by $R_{s}$, and our system can be viewed as a system with $Z_{2}^{(1)} 1$-symmetry. Such a system has a categorical symmetry given by $Z_{1}\left(R_{S}\right)=\mathcal{G}_{Z_{2}^{(1)}}^{3}$, which is a $Z_{2}^{(1)}$ two-gauge theory in $(4+1) \mathrm{D}[14,17,68-73]$. The categorical symmetry contains $Z_{2} 1$-symmetry from the fusion of the stringlike $Z_{2}^{(1)}$ charges in the $4+1 \mathrm{D} Z_{2}^{(1)}$ twogauge theory. The categorical symmetry also contains $\widetilde{Z}_{2}^{(1)}$ 1 -symmetry. The charge of $\widetilde{Z}_{2}^{(1)}$ symmetry is the stringlike $Z_{2}^{(1)}$ flux in the $4+1 \mathrm{D} Z_{2}^{(1)}$ two-gauge theory. The condensation of the stringlike $Z_{2}^{(1)}$ charges induces a confinement transition from the $Z_{2}$ topological order to trivial order. The critical point of the confinement transition has the $Z_{2}^{(1)} \vee \widetilde{Z}_{2}^{(1)}$ categorical symmetry.

Such a critical point with $Z_{2}^{(1)} \vee \widetilde{Z}_{2}^{(1)}$ categorical symmetry can be realized by a model on a cubic lattice with a spin $1 / 2$ living on each link. We label the sites by $i$ and links by $i j$. The Hamiltonian of our model is given by

$$
\begin{aligned}
H= & -B \sum_{\langle i j\rangle} Z_{i j}-J \sum_{\langle i j k l\rangle} X_{i j} X_{j k} X_{k l} X_{l i} \\
& +U \sum_{i}\left(1-\prod_{j \text { next to } i} Z_{i j}\right),
\end{aligned}
$$

where $\sum_{i}$ sums over all sites, $\sum_{\langle i j\rangle}$ over all links, and $\sum_{\langle i j k l\rangle}$ over all squares of the cubic lattice. We also assume $U \stackrel{\langle i j k l\rangle}{\rightarrow}$ $+\infty$ and $B, J>0$.

When $B=0$ and $J>0$, the above model $H$ has a ground state with a $Z_{2}$ topological order. When $B>0$ and $J=0$, the model has a trivial product state $\left|\left\{Z_{i j}=1\right\}\right\rangle$ as its ground state and is in the trivial phase (the confined phase). Changing 
from $(B=0, J>0)$ to $(B>0, J=0)$ induces a confinement transition.

The model explicitly has a $Z_{2}^{(1)} 1$-symmetry generated by the following membrane operator:

$$
C_{Z_{2}^{(1)}}\left(\tilde{M}^{2}\right)=\prod_{\langle i j\rangle \in \widetilde{M}^{2}} Z_{i j} .
$$

The membrane operator $C_{Z_{2}^{(1)}}\left(\tilde{M}^{2}\right)$ acts on a membrane $\tilde{M}^{2}$ in the dual cubic lattice, where the membrane $\tilde{M}^{2}$ is formed by the squares of the dual lattice. Since the squares of the dual lattice one-to-one correspond to the links in the original cubic lattice, the membrane $\widetilde{M}^{2}$ is formed by the links of the original lattice. $\prod_{\langle i j\rangle \in \widetilde{M}^{2}}$ is a product over all the links from $\widetilde{M}^{2}$. Such a $Z_{2}^{(1)} 1$-symmetry is spontaneously broken in the phase with $Z_{2}$ topological order, and is unbroken in the trivial phase (the confined phase).

To see the $\widetilde{Z}_{2}^{(1)} 1$-symmetry explicitly, we need to do a duality transformation to obtain a new model with a spin $1 / 2$ living on each square face $\langle i j k l\rangle$ of the cubic lattice. Let $\widetilde{X}_{i j k l}, \widetilde{Y}_{i j k l}$, and $\widetilde{Z}_{i j k l}$ be the Pauli operators acting on the spin $1 / 2$ on face $\langle i j k l\rangle$. The duality mapping is given by

$$
\begin{aligned}
X_{i j} X_{j k} X_{k l} X_{l i} & \rightarrow \tilde{X}_{i j k l}, \quad Z_{i j} \rightarrow \prod_{k, l \text { next to } i, j} \widetilde{Z}_{i j k l}, \\
\prod_{\langle i j k l\rangle \in c} \tilde{X}_{i j k l} & =1
\end{aligned}
$$

where $\prod_{\langle i j k l\rangle \in c}$ is the product over all the six faces of a cube $c$. The dual Hamiltonian is given by

$$
\begin{aligned}
\widetilde{H}= & -B \sum_{\langle i j\rangle} \prod_{k, l \text { next to } i, j} \widetilde{Z}_{i j k l}-J \sum_{\langle i j k l\rangle} \widetilde{X}_{i j k l} \\
& +\widetilde{U} \sum_{c}\left(1-\prod_{\langle i j k l\rangle \in c} \tilde{X}_{i j k l}\right),
\end{aligned}
$$

where $\widetilde{U} \rightarrow \infty$.

When $B>0$ and $J=0$, the above dual model $\tilde{H}$ has a ground state with a dual $\widetilde{Z}_{2}$ topological order [which corresponds to the confined phase with the trivial $Z_{2}$ topological order in the original model (103)]. When $B=0$ and $J>0$, the model has a trivial product state $\left|\left\{\widetilde{X}_{i j k l}=1\right\}\right\rangle$ as its ground state and is in the dual trivial phase [which corresponds to the phase with the $Z_{2}$ topological order in the original model (103)]. Changing from $(B>0, J=0)$ to $(B=0, J>$ 0 ) induces a dual confinement transition, while changing from $(B=0, J>0)$ to $(B>0, J=0)$ induces a confinement transition.

The dual model $\widetilde{H}$ explicitly has a $\widetilde{Z}_{2}^{(1)} 1$-symmetry generated by the following membrane operator:

$$
C_{\widetilde{Z}_{2}^{(1)}}\left(M^{2}\right)=\prod_{\langle i j k l\rangle \in M^{2}} \widetilde{X}_{i j k l} .
$$

The membrane operator $C_{\widetilde{Z}_{2}^{(1)}}\left(M^{2}\right)$ acts on a membrane $M^{2}$ which is formed by the squares of the lattice. Such a $\widetilde{Z}_{2}^{(1)}$ 1 -symmetry is spontaneously broken in the phase with dual

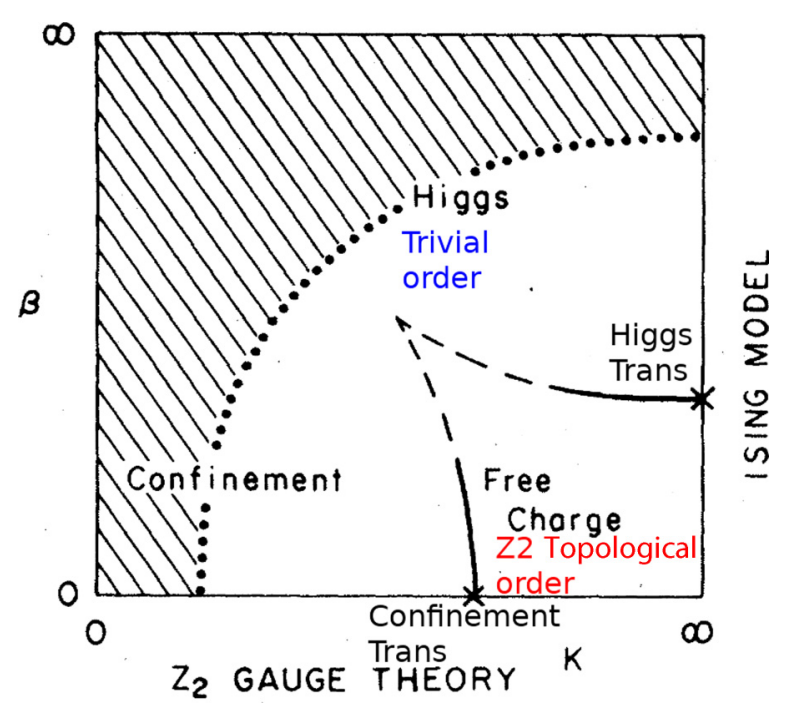

FIG. 5. The phase diagram of Eq. (108).

$\widetilde{Z}_{2}$ topological order (the confined phase with the trivial $Z_{2}$ topological order), and is unbroken in the dual trivial phase (the phase with the $Z_{2}$ topological order). If the confinement transition from the $\widetilde{Z}_{2}$ topological order to the trivial order $i$ given by a single critical point, then such a gapless critical state will have the full $Z_{2}^{(1)} \vee \widetilde{Z}_{2}^{(1)}$ categorical symmetry which is not spontaneously broken.

It is well known that there are two ways to go from $3+1 \mathrm{D}$ $Z_{2}$ topological order (i.e., $Z_{2}$ gauge theory) to trivial order, either via Higgs condensation or via confinement. Reference [74] studied a model defined by the $3+1 \mathrm{D}$ path integral for the following action:

$$
\begin{aligned}
S & =\frac{K}{2} \sum_{\langle i j k l\rangle} \sigma_{i j} \sigma_{j k} \sigma_{k l} \sigma_{l i}+\frac{\beta}{2} \sum_{i j} \phi_{i}^{\dagger} \sigma_{i j} \phi_{j}, \\
\sigma_{i j} & = \pm 1, \quad \phi_{i}= \pm 1 .
\end{aligned}
$$

The model has $Z_{2}$ topological order when $\beta \sim 0$ and $K \sim$ $+\infty$. The model has trivial order when $\beta \sim+\infty$ or $K \sim 0$ (see Fig. 5).

When $K=+\infty$, as we change $\beta$ from zero to $+\infty$, the model goes through a second-order Ising transition (i.e., Higgs transition) described by a critical point. When $\beta=0$, as we change $K$ from zero to $+\infty$, the model goes through a first-order confinement transition [75]. Let us assume that we can modify the model to make the confinement transition to be a continuous transition described by a critical point. One may wonder whether the two critical points for the two transitions are the same or not.

Our above discussions suggest that the two critical points are different since they have different categorical symmetries. One has $Z_{2} \vee Z_{2}^{(2)}$ categorical symmetry and the other has $Z_{2}^{(1)} \vee \widetilde{Z}_{2}^{(1)}$ categorical symmetry, and $Z_{1}\left[3 \mathcal{R} \operatorname{ep}\left(Z_{2}\right)\right]$ are different from $Z_{1}\left(3 \mathcal{V} \mathrm{ec}_{Z_{2}^{(1)}}\right)$. Thus categorical symmetries deepen our understanding of the Higgs transition and the confinement transition, as well as their relationship. 
(a)

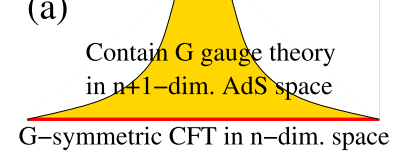

FIG. 6. (a) A CFT with zero symmetry $G$ is described by a AdS/CFT dual that contains a $G$ gauge theory in the AdS space in one higher dimension. (b) The CFT at the transition of spontaneously $G$ symmetry-breaking has a categorical symmetry $\mathcal{G}_{G}^{n}=$ $G \vee G^{(n-1)}$ described by excitations in a $G$-gauge theory in one higher dimension.

\section{SUMMARY}

In this paper, we show that a quantum system in $n$ dimensional space with a zero symmetry $G$ actually has a larger symmetry, which includes both the zero symmetry $G$ and an algebraic $(n-1)$-symmetry, denoted as $G^{(n-1)}$, the transformations of which are given by Wilson loop operators. In fact, the $G$-symmetric quantum system actually has a larger categorical symmetry characterized by a braided fusion $n$ category $\mathcal{G}_{G}^{n}$. We also denote the categorical symmetry $\mathcal{G}_{G}^{n}$ as $G \vee G^{(n-1)}$, since it includes both the zero symmetry $G$ and the algebraic $(n-1)$-symmetry $G^{(n-1)}$.

We find that any gapped state in a system with a categorical symmetry must partially (and only partially) break the categorical symmetry spontaneously. However, for the gapless critical state at the transition of the spontaneous $G$ symmetry breaking, the categorical symmetry $G \vee G^{(n-1)}$ is not broken. In particular, the critical state has both the zero symmetry $G$ and the algebraic $(n-1)$-symmetry $G^{(n-1)}$.

It was proposed that a CFT in $n$-dimensional space with zero symmetry $G$ has a AdS/CFT dual that contains a $G$ gauge theory in the $(n+1) \mathrm{d}$ AdS space [see Fig. 6(a)] [32-35]. If the AdS/CFT dual contains only a $G$-gauge theory (with the fluctuations of both gauge charges and gauge flux) and gravity in the $(n+1) \mathrm{d}$ AdS space [see Fig. 6(a)], then the corresponding CFT in $n$-dimensional space must be a particular one with zero symmetry $G$. But there are many CFT's with zero symmetry $G$. Which is the right one? The result in this paper [see Fig. 6(b)] suggests that it is the CFT at the transition of the spontaneous $G$ symmetry breaking that is dual to a theory that contains only a $G$-gauge theory and gravity in the $(n+1) \mathrm{d}$ AdS space. This is because the specific CFT has the categorical symmetry $G \vee G^{(n-1)}$, the excitation of which matches that of the dual theory in the AdS bulk. In other words, Fig. 6(a) corresponds to the CFT boundary in Fig. 6(b). This is demonstrated in Fig. 7. The categorical symmetry of a CFT can help us to select the AdS/CFT dual of the CFT, which is an important application of the categorical symmetry.

\section{ACKNOWLEDGMENTS}

We would like to thank Liang Kong, Tian Lan, Shu-heng Shao, and Hao Zheng for many helpful discussions. This research was partially supported by NSF Grant No. DMS1664412. This work was also partially supported by the

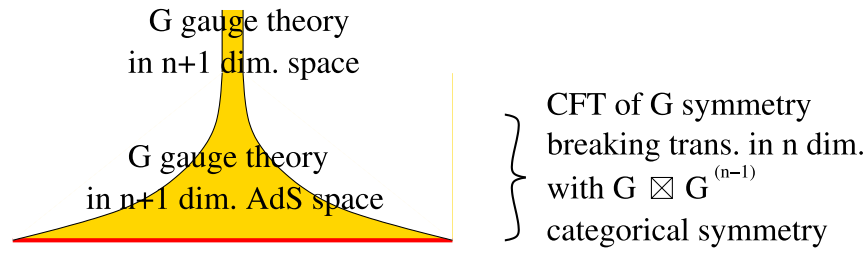

FIG. 7. A combination of the holographic picture for AdS/CFT duality and the holographic picture for emergent categorical symmetry in the CFT for the $G$ symmetry-breaking transition.

Simons Collaboration on Ultra-Quantum Matter, a grant from the Simons Foundation (Grant No. 651440).

\section{APPENDIX A: LIST OF TERMINOLOGIES}

Here, we explain some mathematical terminologies used in this paper, at the level of physical rigorousness. An ordinary category has objects and morphisms (also called 1-morphisms), and it is also called a 1-category. A 2-category generalizes this by also including 2-morphisms between the 1 -morphisms. Continuing this up to $n$-morphisms between $(n-1)$-morphisms gives an $n$-category.

In condensed-matter physics, a n-category can correspond to a collection of topological orders in $n \mathrm{D}$ spacetime. The topological orders in the collection correspond to the simple objects in the category. The composite objects correspond to degenerate states where several different topological orders happen to have the same energy. The gapped domain walls between different topological orders correspond to one morphisms between different simple objects. The gapped domain walls within the same topological order correspond to one morphisms between the same simple object. The domain walls (1-morphisms) can also have domain walls with one lower dimension between them, which correspond to two morphisms, etc. In general, a $m$-morphism that connects the trivial $(m-1)$-morphism to itself corresponds to a codimension- $m$ excitation. A $m$-morphism that connects a nontrivial $(m-1)$-morphism to itself corresponds to a domain wall on the codimension- $(m-1)$ excitation. A $m$ morphism that connects two $(m-1)$-morphisms corresponds to a domain wall between the two codimension- $(m-1)$ excitations. A $n$-morphism corresponds to an "instanton" in spacetime (i.e., an insertion of a local operator in spacetime). A $(n-1)$-morphism corresponds to a pointlike excitation.

Two topological orders can be stacked to form the third topological order. Under the stacking operation, topological orders form a monoid (which is similar to a group, but without the need for an inverse operation) [30]. If we include the stacking operation in the $n$-category, the $n$-category will become a fusion n-category. Thus a collection of topological orders in $n$-dimensional spacetime is actually described by a monoidal $n$-category.

The excitation in a single topological order in $n \mathrm{D}$ spacetime is described by a fusion $(n-1)$-category. Now the objects in the fusion $(n-1)$-category correspond to codimension-1 excitations (domain walls). The 
1-morphisms correspond to codimension-2 excitations (domain walls on domain walls), etc.

An example of a fusion 1-category is $\mathcal{V e c}$. The objects in $\mathcal{V}$ ec are the vector spaces. The simple objects are the (equivalent classes of) one-dimensional vector spaces and composite objects are multidimensional vector spaces. There is only one simple object. We see that composite objects are direct sums of simple objects. The morphisms (the 1-morphisms) correspond to the linear operators acting on the vector spaces. The tensor product of the vector spaces defines the fusion of the objects. We see that one-dimensional vector space is the unit of the tensor product, and hence the simple object is the unit of the fusion. We also call such a fusion unit as the trivial object. The 1-morphism that connects the simple object to itself is proportional to the one-by-one identity operator, and thus is also trivial. So we refer to fusion 1-category $\mathcal{V e c}$ as a trivial category. We also have a trivial higher category, which has only one simple nondescendant morphism (which is not condensation of other excitations $[7,8,60,61]$ ) at every level. We denote the trivial higher category as $n \mathcal{V e c}$.

Another example of a fusion $n$-category is $\operatorname{Rep}(G)$. The $(n-1)$-morphisms in $\mathcal{R e p}(G)$ are the representations of the group $G$ which correspond to pointlike excitations in $n$ dimensional space. The tensor product of the $G$ representations defines the fusion of the $(n-1)$-morphisms. The simple $(n-1)$-morphisms are the (equivalent classes of) irreducible representations and composite objects are the reducible representations. The reducible representations are direct sums of irreducible representations, and thus composite morphisms are direct sums of simple morphisms. The $n$-morphisms correspond to the symmetric operators acting on group representations.

All the 1-morphisms and two morphisms on the trivial object in a fusion $n$-category form a braided fusion $(n-1)$-category. It can be used to describe codimension2 and higher excitations in an anomaly-free topological order (after dropping the codimension-1 excitations which are always descendent for anomaly-free topological orders).

We also need a notion of a local fusion n-category: A fusion $n$-category $\mathcal{F}$ is local if we can add morphisms in a consistent way, such that all the resulting simple morphisms are isomorphic to the trivial one. Physically, the process of "adding morphisms" corresponds to explicit breaking of the (algebraic higher) symmetry. This is because $\mathcal{F}$ only has morphisms that correspond to symmetric operators. Adding morphisms means including morphisms that correspond to symmetry-breaking operators. If after breaking all the symmetry $\mathcal{F}$ becomes a trivial product phase of bosons or fermions, then $\mathcal{F}$ is a local fusion $n$-category.

\section{APPENDIX B: PROOF OF EQUIVALENCE OF THE 1D (PROJECTED) $Z_{2}$ MINIMALLY COUPLED MODEL AND THE (PROJECTED) ISING MODEL}

We are going to prove that the (projected) minimally coupled model Eq. (21), the (projected) Ising model Eq. (10), and the (projected) dual Ising model Eq. (11) are all equivalent. In this subsection, we always consider the projected sub-Hilbert space.

The strategy is to show that in the minimally coupled model the Hamiltonian is built from the logical operators within a stabilized subspace. We begin with $2 N$ physical qubits on $2 N$ sites. We take those states stabilized by $Z_{2 i-1} Z_{2 i} Z_{2 i+1}$, for $i=1, \ldots, N$. This is to constrain ourselves to states $Z_{2 i-1} Z_{2 i} Z_{2 i+1}=1$.

We first show that the minimally coupled model is equivalent to the Ising model. The proof uses the stabilized code formalism in quantum information (QI).

For simplicity, we switch the notation in the minimal coupled model to that in QI, $X_{i} \rightarrow X_{2 i-1}, \widetilde{X}_{i+\frac{1}{2}} \rightarrow X_{2 i}$, and similarly for the Pauli $Z$ 's. In this way, the low-energy constraints in the limit $U \rightarrow \infty$ are mapped to the stabilizers. And the Hamiltonian becomes

$$
H=-B \sum_{i} X_{2 i} X_{2 i+1} X_{2 i+2}-J \sum_{i} Z_{2 i},
$$

acting on the $2 N$ number of physical qubits. And there are then in total $2^{N}$ logical qubits.

First, we show

$$
\mathcal{X}_{i}=X_{2 i} X_{2 i+1} X_{2 i+2}, \quad \mathcal{Z}_{i}=Z_{2 i+1}
$$

are the Pauli- $X$ and Pauli- $Z$ operators on the logical qubits. (And $\mathcal{X}_{N}=X_{2 N} X_{1} X_{2}, \mathcal{Z}_{N}=Z_{1}$.) Obviously, they satisfy the algebra of the Pauli matrices, and each of them commute with the stabilizers. Since there are $N$ Pauli- $Z$ operators we find that their eigenvalue labels are in one-to-one correspondence to the states in the stabilized space. Therefore, these logical Pauli operators $\left\{\mathcal{X}_{i}, \mathcal{Z}_{i} \mid i=1, \ldots, N\right\}$ form a complete basis for all operators acting on the logic qubits.

Then we find that $\mathcal{Z}_{i} \mathcal{Z}_{i+1}=Z_{2 i+2}\left(\right.$ and $\left.\mathcal{Z}_{N} \mathcal{Z}_{1}=Z_{2}\right)$ are the same as a pair of Pauli- $Z$ operators on the logical qubits. It follows from (B2) that $\mathcal{Z}_{i} \mathcal{Z}_{i+1}=Z_{2 i+1} Z_{2 i+3}\left(Z_{2 i+1} Z_{2 i+2} Z_{2 i+3}\right)$, where it is a stabilizer that we multiply on in the bracket. The model $^{11}$ in terms of logical operators becomes

$$
H=-B \sum_{i} \mathcal{X}_{i}-J \sum_{i} \mathcal{Z}_{i-1} \mathcal{Z}_{i}
$$

Finally, the global symmetry operator $\prod_{i} X_{2 i+1}$ in the minimally coupled model also maps to $\prod_{i} \mathcal{X}_{i}$, the global $Z_{2}$ symmetry operator on the logical qubits. We may, of course, add this operator initially to the set of stabilizers and the analysis remains the same but with $2^{N-1}$ logical qubits.

There is a caveat in this proof. If different models can be mapped to the same Hamiltonian on the same set of logical qubits defined in a stabilized space, they are indeed the same at the algebraic level. However, we need to further check if they have the same "locality property." Even though it is hard to define the property generically, for the one-dimensional models we study here, they all have the properties that neighboring logical Pauli operators come from neighboring local terms composed of physical Pauli operators.

\footnotetext{
${ }^{11}$ This is not a Hamiltonian built of stabilizers, but one built from logical Pauli operators within the stabilized subspace.
} 


\section{APPENDIX C: NON-ON-SITE $Z_{2}$ SYMMETRY TRANSFORMATIONS}

The non-on-site $Z_{2}$ symmetry transformation $U=$ $\prod_{i} X_{i} \prod_{i} s_{i-\frac{1}{2}, i+\frac{1}{2}}$ [where $s_{i j}$ is given in Eq. (68)] transforms $X_{i}$ in the following way:

$$
\begin{aligned}
\left(\prod_{j} X_{j} \prod_{j} s_{j, j+1}\right) X_{i}\left(\prod_{j} X_{j} \prod_{j} s_{j, j+1}\right) \\
=\frac{1-Z_{i-1}+Z_{i}+Z_{i-1} Z_{i}}{2} \frac{1-Z_{i}+Z_{i+1}+Z_{i} Z_{i+1}}{2} X_{i} \\
\quad \times \frac{1-Z_{i-1}+Z_{i}+Z_{i-1} Z_{i}}{2} \frac{1-Z_{i}+Z_{i+1}+Z_{i} Z_{i+1}}{2}
\end{aligned}
$$

$$
\begin{aligned}
= & \frac{1-Z_{i-1}+Z_{i}+Z_{i-1} Z_{i}}{2} \frac{1-Z_{i}+Z_{i+1}+Z_{i} Z_{i+1}}{2} \\
& \times \frac{1-Z_{i-1}-Z_{i}-Z_{i-1} Z_{i}}{2} \frac{1+Z_{i}+Z_{i+1}-Z_{i} Z_{i+1}}{2} X_{i} \\
= & \left(\frac{1-Z_{i-1}+Z_{i}+Z_{i-1} Z_{i}}{2} \frac{1-Z_{i-1}-Z_{i}-Z_{i-1} Z_{i}}{2}\right) \\
& \times\left(\frac{1-Z_{i}+Z_{i+1}+Z_{i} Z_{i+1}}{2} \frac{1+Z_{i}+Z_{i+1}-Z_{i} Z_{i+1}}{2}\right) X_{i} \\
= & \frac{\left(1-Z_{i-1}\right)-\left(1+Z_{i-1}\right)}{2} \frac{\left(1+Z_{i+1}\right)-\left(1-Z_{i+1}\right)}{2} X_{i} \\
= & -Z_{i-1} X_{i} Z_{i+1} .
\end{aligned}
$$

In other words,

$$
X_{i} \leftrightarrow-Z_{i-1} X_{i} Z_{i+1}
$$

[1] L. D. Landau, Phys. Z. Sowjetunion 11, 26 (1937).

[2] L. D. Landau, Phys. Z. Sowjetunion 11, 545 (1937).

[3] P. Ginsparg, arXiv:hep-th/9108028 (1991).

[4] P. Di Francesco, P. Mathieu, and D. Sénéchal, Conformal Field Theory (Springer, New York, 1997).

[5] T. Lan, L. Kong, and X.-G. Wen, Phys. Rev. X 8, 021074 (2018).

[6] T. Lan and X.-G. Wen, Phys. Rev. X 9, 021005 (2019).

[7] L. Kong, T. Lan, X.-G. Wen, Z.-H. Zhang, and H. Zheng, arXiv:2003.08898 (2020).

[8] L. Kong, T. Lan, X.-G. Wen, Z.-H. Zhang, and H. Zheng, arXiv:2005.14178 (2020).

[9] J. Frölich, J. Fuchs, I. Runkel, and C. Schweigert, Nucl. Phys. B 763, 354 (2007).

[10] A. Davydov, L. Kong, and I. Runkel, Adv. Theor. Math. Phys. 15, 43 (2011).

[11] C.-M. Chang, Y.-H. Lin, S.-H. Shao, Y. Wang, and X. Yin, J. High Energy Phys. 01 (2019) 26.

[12] R. Thorngren and Y. Wang, arXiv:1912.02817 (2019).

[13] L. Kong, W. Yuan, and H. Zheng, arXiv:1912.13168 (2019).

[14] A. Kapustin and R. Thorngren, arXiv:1309.4721 (2013).

[15] D. Gaiotto, A. Kapustin, N. Seiberg, and B. Willett, J. High Energy Phys. 02 (2015) 172.

[16] R. Thorngren and C. von Keyserlingk, arXiv:1511.02929 (2015).

[17] R. Costa de Almeida, J. P. Ibieta-Jimenez, J. Lorca Espiro, and P. Teotonio-Sobrinho, arXiv:1711.04186 (2017)

[18] R. Kobayashi, K. Shiozaki, Y. Kikuchi, and S. Ryu, Phys. Rev. B 99, 014402 (2019).

[19] E. Lake, arXiv:1802.07747 (2018).

[20] X.-G. Wen, Phys. Rev. B 99, 205139 (2019).

[21] Z. Wan and J. Wang, Phys. Rev. D 99, 065013 (2019).

[22] Z. Wan and J. Wang, Ann. Math. Sci. Appl. 4, 107 (2018).

[23] A. Kitaev, Ann. Phys. (NY) 303, 2 (2003).

[24] X.-G. Wen, Phys. Rev. Lett. 90, 016803 (2003).

[25] M. Levin and X.-G. Wen, Phys. Rev. B 67, 245316 (2003).

[26] Z. Nussinov and G. Ortiz, Proc. Natl. Acad. Sci. USA 106, 16944 (2009).
[27] Z. Nussinov and G. Ortiz, Ann. Phys. (NY) 324, 977 (2009).

[28] B. Yoshida, Ann. Phys. (NY) 326, 15 (2011).

[29] H. Bombín, Commun. Math. Phys. 327, 387 (2014).

[30] L. Kong and X.-G. Wen, arXiv:1405.5858 (2014)

[31] W. Ji and X.-G. Wen, Phys. Rev. Research 1, 033054 (2019).

[32] J. Maldacena, Adv. Theor. Math. Phys. 2, 231 (1998).

[33] E. Witten, Adv. Theor. Math. Phys. 2, 253 (1998).

[34] I. R. Klebanov and E. Witten, Nucl. Phys. B 556, 89 (1999).

[35] D. Harlow and H. Ooguri, arXiv:1810.05338 (2018).

[36] A. Belavin, A. Polyakov, and A. Zamolodchikov, Nucl. Phys. B 241, 333 (1984).

[37] A. Zamolodchikov and V. Fateev, Sov. Phys. JETP 62, 215 (1985).

[38] R. Verresen, R. Thorngren, N. G. Jones, and F. Pollmann, arXiv:1905.06969 (2019).

[39] W. Ji, S.-H. Shao, and X.-G. Wen, Phys. Rev. Research 2, 033317 (2020).

[40] M. Levin, Commun. Math. Phys. 378, 1081 (2020).

[41] D. Harlow and H. Ooguri, Phys. Rev. Lett. 122, 191601 (2019).

[42] S. Doplicher, Commun. Math. Phys. 85, 73 (1982).

[43] S. Doplicher and R. Longo, Commun. Math. Phys. 88, 399 (1983).

[44] D. Buchholz, S. Doplicher, and R. Longo, Ann. Phys. (NY) 170 , 1 (1986).

[45] F. Verstraete and J. I. Cirac, arXiv:cond-mat/0407066 (2004).

[46] G. Vidal, Phys. Rev. Lett. 99, 220405 (2007).

[47] M. Levin and C. P. Nave, Phys. Rev. Lett. 99, 120601 (2007).

[48] Z.-C. Gu and X.-G. Wen, Phys. Rev. B 80, 155131 (2009).

[49] X. Chen, Z.-C. Gu, Z.-X. Liu, and X.-G. Wen, Science 338, 1604 (2012).

[50] W.-Q. Chen, C.-M. Jian, L. Kong, Y.-Z. You, and H. Zheng, Phys. Rev. B 102, 045139 (2020).

[51] L. Kong and H. Zheng, J. High Energ. Phys. 02 (2020) 150.

[52] L. Kong and H. Zheng, arXiv:1912.01760 (2019).

[53] R. Dijkgraaf and E. Witten, Commun. Math. Phys. 129, 393 (1990).

[54] G. 't Hooft, NATO Adv. Study Inst. Ser. B Phys. 59, 135 (1980).

[55] X.-G. Wen, Phys. Rev. D 88, 045013 (2013). 
[56] M. Freedman, C. Nayak, K. Shtengel, K. Walker, and Z. Wang, Ann. Phys. (NY) 310, 428 (2004).

[57] M. A. Levin and X.-G. Wen, Phys. Rev. B 71, 045110 (2005).

[58] X. Chen, Z.-X. Liu, and X.-G. Wen, Phys. Rev. B 84, 235141 (2011).

[59] S. Gukov and A. Kapustin, arXiv:1307.4793 (2013).

[60] D. Gaiotto and T. Johnson-Freyd, arXiv:1905.09566 (2019).

[61] T. Johnson-Freyd, arXiv:2003.06663 (2020).

[62] T. Lan, L. Kong, and X.-G. Wen, Phys. Rev. B 95, 235140 (2017).

[63] D. S. Freed and C. Teleman, arXiv:1806.00008 (2018).

[64] L. Kong, X.-G. Wen, and H. Zheng, arXiv:1502.01690 (2015).

[65] L. Kong, X.-G. Wen, and H. Zheng, Nucl. Phys. B 922, 62 (2017).

[66] N. Read and S. Sachdev, Phys. Rev. Lett. 66, 1773 (1991).
[67] X. G. Wen, Phys. Rev. B 44, 2664 (1991).

[68] J. C. Baez and U. Schreiber, arXiv:math/0511710 (2007).

[69] F. Girelli, H. Pfeiffer, and E. M. Popescu, J. Math. Phys. 49, 032503 (2008).

[70] J. C. Baez and J. Huerta, Gen. Relativ. Gravit. 43, 2335 (2010).

[71] A. Bullivant, M. Calçada, Z. Kádár, P. Martin, and J. F. Martins, Phys. Rev. B 95, 155118 (2017).

[72] A. Bullivant, M. Calcada, Z. Kádár, P. Martin, and J. F. Martins, Rev. Math. Phys. 32, 2050011 (2020).

[73] C. Zhu, T. Lan, and X.-G. Wen, Phys. Rev. B 100, 045105 (2019).

[74] E. Fradkin and S. H. Shenker, Phys. Rev. D 19, 3682 (1979).

[75] M. Creutz, L. Jacobs, and C. Rebbi, Phys. Rev. D 20, 1915 (1979).

[76] C. Gattringer, arXiv:1401.7788 (2014). 